\title{
Indole Derivatives Maintain the Status Quo Between Beneficial Biofilms and Their Plant Hosts
}

\author{
Hadas Ganin, ${ }^{1}$ Natalie Kemper, ${ }^{1}$ Sagit Meir, ${ }^{2}$ Ilana Rogachev, ${ }^{2}$ Shir Ely, ${ }^{1}$ Hassan Massalha, ${ }^{2}$ \\ Aviad Mandaby, ${ }^{3}$ Abraham Shanzer, ${ }^{4}$ Alona Keren-Paz, ${ }^{1}$ Michael M. Meijler, ${ }^{3}$ Sergey Malitsky, ${ }^{5}$ \\ Asaph Aharoni, ${ }^{2}$ and Ilana Kolodkin-Gal', \\ ${ }^{1}$ Department of Molecular Genetics, Weizmann Institute of Science, Rehovot, Israel \\ ${ }^{2}$ Department of Plant and Environmental Sciences, Weizmann Institute of Science \\ ${ }^{3}$ Department of Chemistry, Ben-Gurion University of the Negev, Beer-Sheva, Israel \\ ${ }^{4}$ Department of Chemistry, Weizmann Institute of Science \\ ${ }^{5}$ Metabolic Profiling Unit, Weizmann Institute of Science
}

Accepted 17 February 2019

Biofilms formed by bacteria on plant roots play an important role in maintaining an optimal rhizosphere environment that supports plant growth and fitness. Bacillus subtilis is a potent plant growth promoter, forming biofilms that play a key role in protecting the host from fungal and bacterial infections. In this work, we demonstrate that the development of $B$. subtilis biofilms is antagonized by specific indole derivatives that accumulate during symbiotic interactions with plant hosts. Indole derivatives are more potent signals when the plant polysaccharide xylan serves as a carbon source, a mechanism to sustain beneficial biofilms at a biomass that can be supported by the plant. Moreover, $B$. subtilis biofilms formed by mutants resistant to indole derivatives become deleterious to the plants due to their capacity to consume and recycle plant polysaccharides. These results demonstrate how a dynamic metabolitebased dialogue can promote homeostasis between plant hosts and their beneficial biofilm communities.

Keywords: molecular signaling, microbial ecology, metabolism

Bacterial biofilms are intricate microbial communities encased in self-produced and self-secreted extracellular matrix polymers composed of exopolysaccharides, proteins, and DNA (Branda et al. 2005; Steinberg and Kolodkin-Gal 2015) and often induce the formation of biogenic minerals (Keren-Paz et al. 2018; Li et al. 2015, 2016). Biofilms are widespread in nature and grant their resident cells protection against environmental stresses as well as antimicrobial agents (Bryers 2008; Kolter and Greenberg 2006).

${ }^{\dagger}$ Corresponding author: I. Kolodkin-Gal;

Ilana.kolodkin-gal@weizmann.ac.il

Funding: The Kolodkin-Gal lab is supported by the Israeli Science Foundation (ISF) grant number 119/16, Scholl Center for water and climate, The Kekst Family Institute for Medical Genetics, and the ISF I-CORE grant 152/1. I. Kolodkin-Gal is a recipient of the Rowland and Sylvia Career Development Chair.

*The $e$-Xtra logo stands for "electronic extra" and indicates that 14 supplementary figures and two supplementary tables are published online.

The author(s) declare no conflict of interest.

() 2019 The American Phytopathological Society
The primary model of our study is Bacillus subtilis, a grampositive rhizosphere bacterium (Chen et al. 2013; Vlamakis et al. 2013). Various undomesticated strains of B. subtilis and related species isolated from the soil are known to defend plants from diverse fungal and bacterial pathogens (Emmert and Handelsman 1999; Ongena and Jacques 2008). This protection is proposed to be mediated, in part, by the formation of biofilms (Bais et al. 2004; Raaijmakers et al. 2010). B. subtilis also belongs to the group of plant growth-promoting rhizobacteria, which, when applied to soils, can stimulate plant growth, increase yield, and reduce fungal and bacterial pathogen infections without being pathogenic. The complicated network regulating biofilm formation in $B$. subtilis is fairly well-solved (Mielich-Süss and Lopez 2015). Several subnetworks are integrated to activate or repress extracellular polymer substances (EPS) gene expression and biofilm formation, depending on the environmental conditions (Vlamakis et al. 2013). Phosphorylation of the response regulator SpoOA is central to biofilm formation (Branda et al. 2001). Spo0A-P governs the regulatory pathway for EPS (extracellular polymers) gene expression by controlling the activity of the master regulator SinR (Chu et al. 2006), a repressor of the epsA-O and tapA-sipW-tasA operons encoding for exopolysaccharides synthesis (Branda et al. 2004) and amyloid fibers (Romero et al. 2010), respectively. An additional pathway regulating expression of the EPS operons involves the DegS/DegU, a sensor kinase and a response regulator (Verhamme et al. 2007, 2009). Mutants lacking spoOA and $\operatorname{deg} U$ are defective in colony formation, owing to the lack of EPS production and loss of the surface hydrophobicity protein BslA $(\Delta \operatorname{deg} U)$, whereas $\Delta \sin R$ mutant colonies exhibit a hyperwrinkly phenotype due to constitutive expression of the EPS operons (Chu et al. 2006). Under standard laboratory settings, several cues have been associated with biofilm maturation and assembly, including oxygen deprivation (KolodkinGal et al. 2013), nutrient deprivation (Liu et al. 2015; McLoon et al. 2011; Yona et al. 2013), small molecules (Bucher et al. 2015; Lopez et al. 2009; Townsley et al. 2018), calcium (Mhatre et al. 2017; Oppenheimer-Shaanan et al. 2016), and physical cues (Asally et al. 2012; Cairns et al. 2013; Grau et al. 2015; Rubinstein et al. 2012). In addition, it was shown that plant polysaccharides and malic acid may act as signals to induce biofilm formation (Beauregard et al. 2013; Rudrappa et al. 2008) and that plant secretions can enhance chemotaxis (Allard-Massicotte et al. 2016). 
Here, we tested the hypothesis that symbiosis can be achieved via an active crosstalk between bacteria and host. According to this hypothesis, root-associated beneficial communities will develop up to the point where the host can no longer support the burden of the accumulated bacterial biomass. $B$. subtilis is considered beneficial, as its biofilms provide protection from various fungal and bacterial pathogens. Using it as a model, we investigated a dynamic plant-bacteria crosstalk, mediated by indole derivatives synthesized during host-bacteria interactions. These metabolites limit the biofilm development in B. subtilis and maintain biofilm biomass that can be supported by the plant. When host-derived signals are ignored, $B$. subtilis biofilms feed off the host polysaccharides and biofilm communities become deleterious.

\section{RESULTS}

Indole derivatives may act as a plant signal to limit biofilm formation.

Plant-driven metabolites may serve as potential regulators to synchronize the biofilm biomass with the carrying capacity of

A
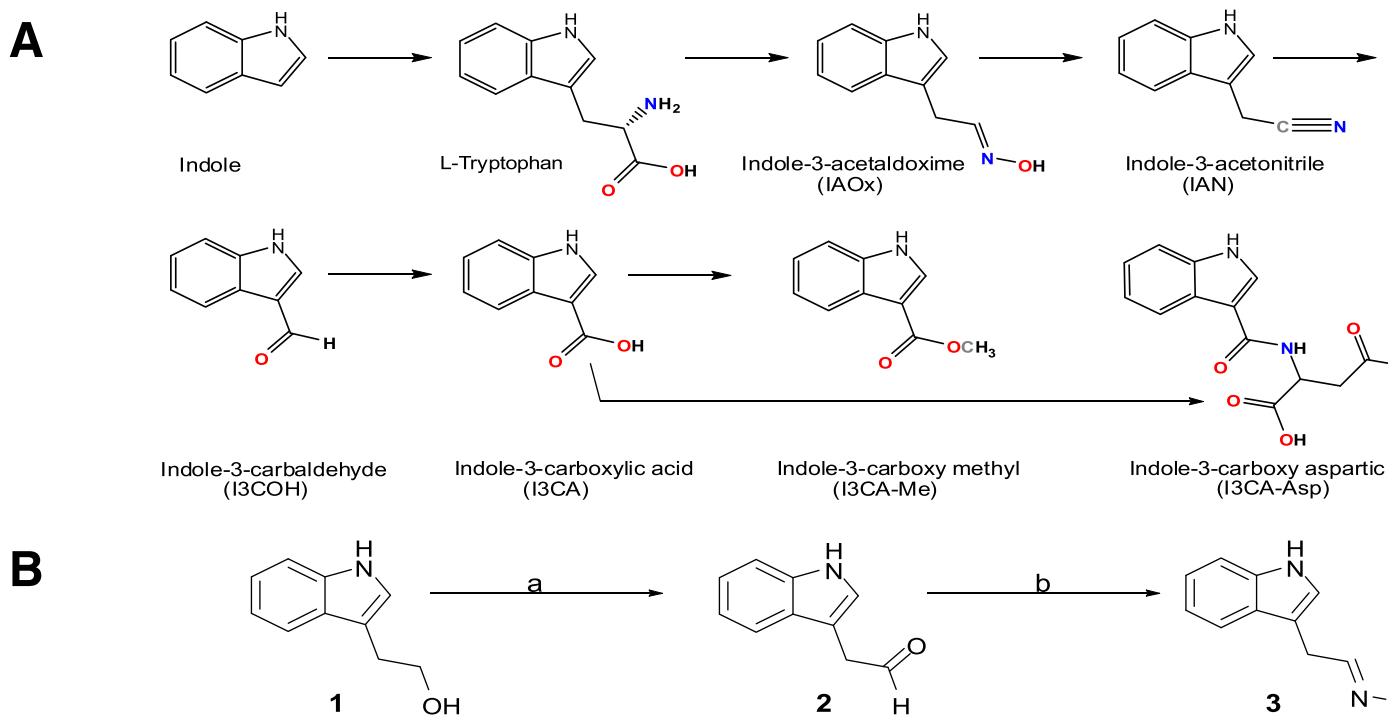

C

C

Indole
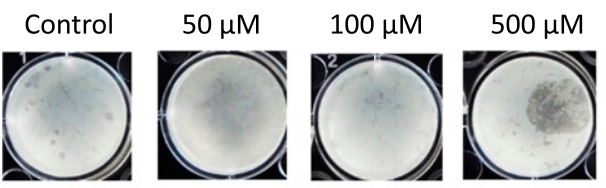

$\operatorname{Trp}$
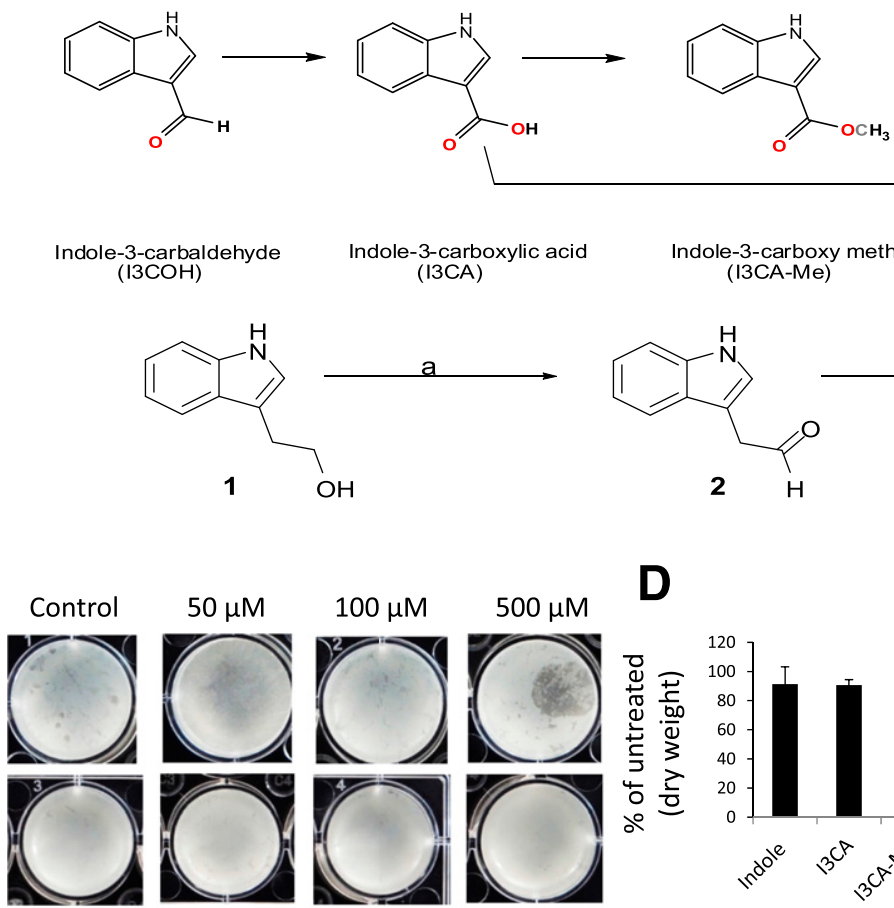

(IAN)

IAOx
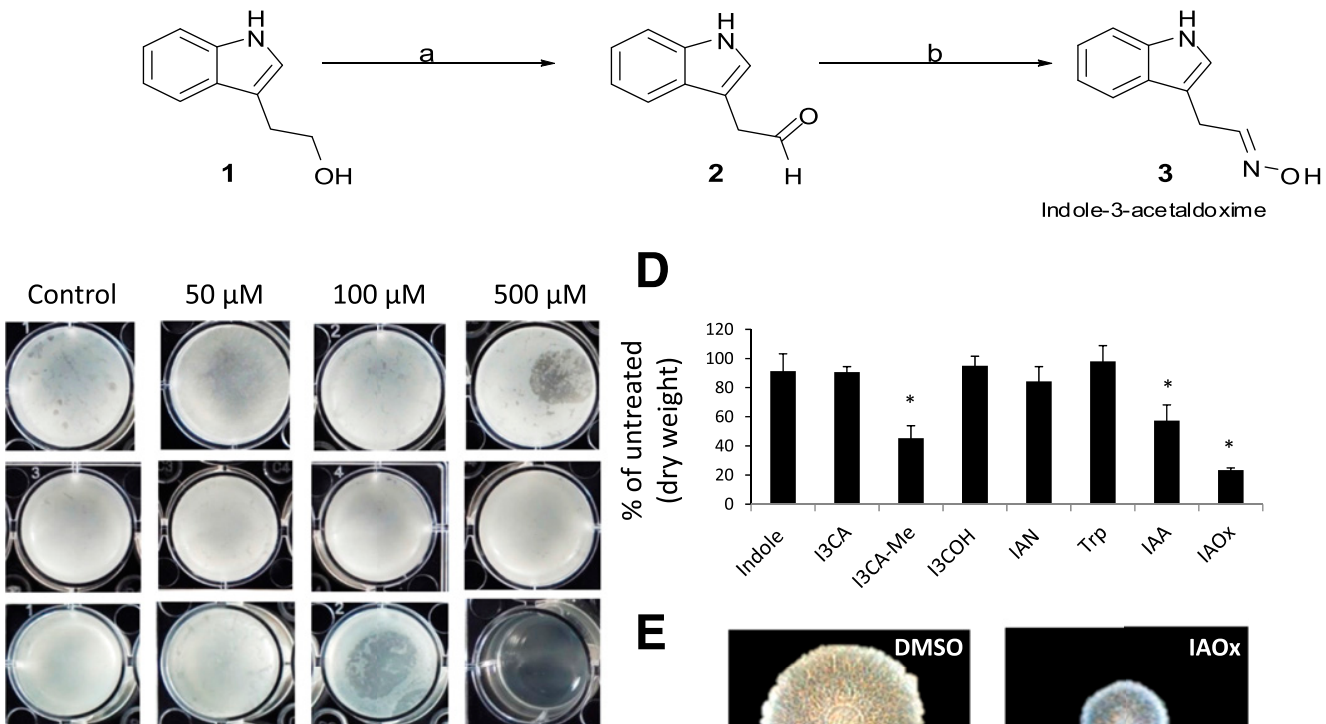

Indole-3-acetaldoxime

IAN
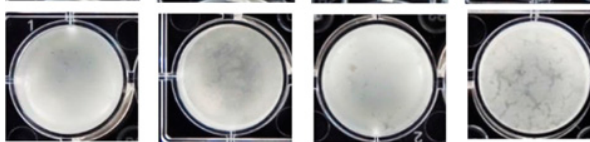

D

$\mathrm{I3COH}$
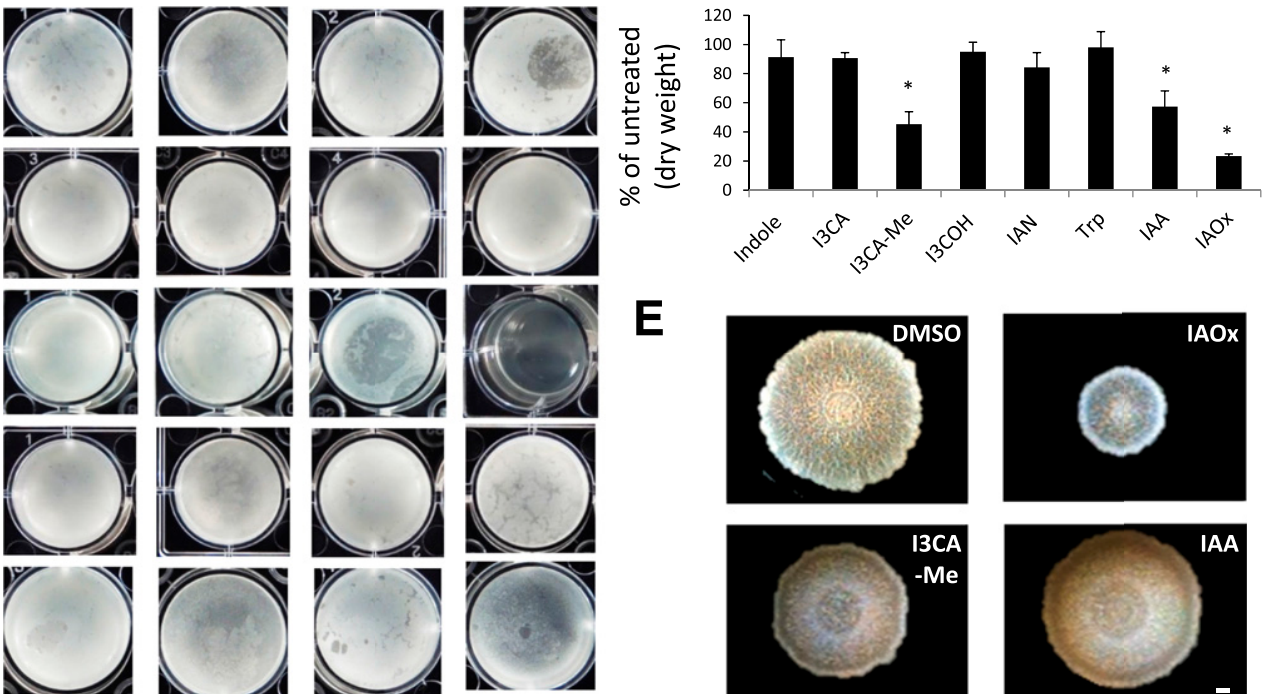

E
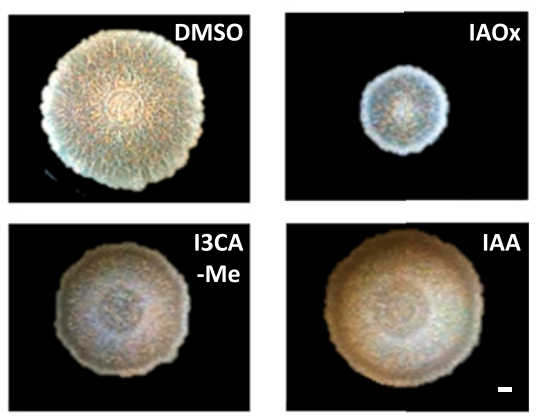

I3CA
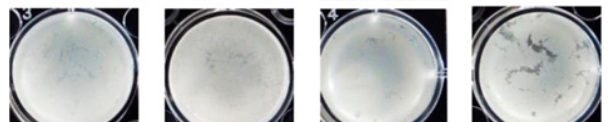

$\mathbf{F}$

I3CA
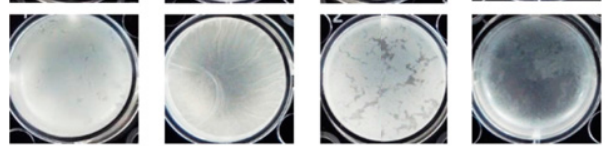

IAA
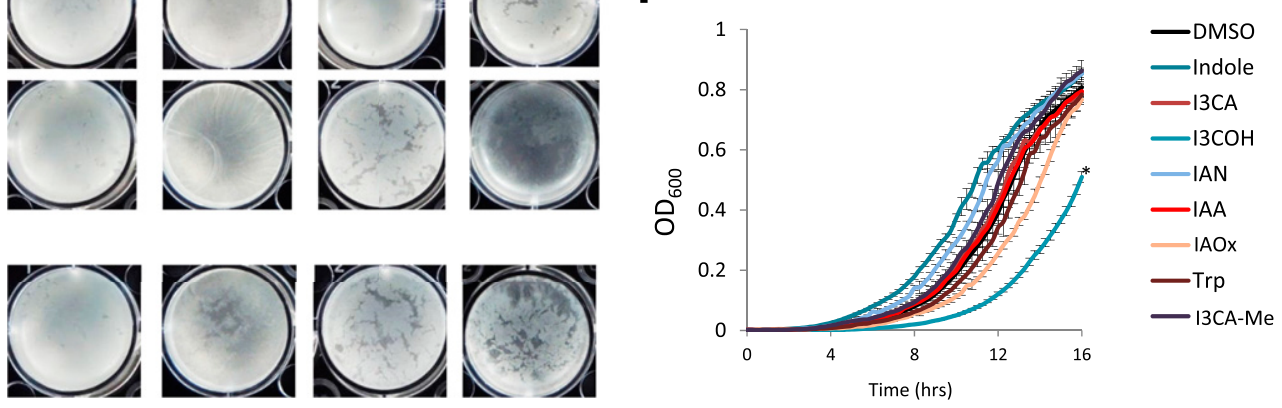
the host. In order to identify the potential regulators of bacterial symbiosis, we focused our efforts on a relatively undomesticated strain of B. subtilis, NCIB 3610, which forms robust and highly structured biofilms both in liquid and on solid medium (Branda et al. 2001) and serves as an efficient biocontrol agent (Chen et al. 2013).

Plants produce various indole derivatives, among them the auxin phytohormone indole-3-acetic acid (IAA), which is reported to be produced and sensed by bacteria (Spaepen et al. 2007). Importantly, plants synthesize numerous root-derived indoles via pathways involved in tryptophan-derived secondary metabolism (Iven et al. 2012). Little is known about the effects of root-derived indoles on the symbiosis of plants with B. subtilis. As indole was found to inhibit biofilm formation throughout bacterial species (Tomberlin et al. 2017), we hypothesized that either indole or metabolites similar to indole can serve to regulate the development of $B$. subtilis biofilms in accordance with the carrying capacity of its plant host.

We decided to take a comprehensive approach and examine several potential structures of root-derived indoles, focusing on representative indole derivatives produced from tryptophan in one biosynthetic pathway (Iven et al. 2012) (Fig. 1A). Two molecules in these pathways were commercially unavailable and, thus, were synthesized. The synthesis of (1E)-N-hydroxy2-(1H-indol-3-yl)ethanimine (indole-3-acetaldoxime [IAOx]) was completed using a combination of two previously reported procedures (Fig. 1B) (Pedras et al. 2002; Sugawara et al. 2009): Commercial indole-3-ethanol (diagram 1 in Figure 1B), was oxidized following Parikh-Doering reaction, and the resulting indole-3-acetaldehyde (diagram 2 in Figure 1B) was then oximated using hydroxylamine hydrochloride and sodium acetate in $\mathrm{EtOH}$ to afford oxime (diagram 3 in Figure 1B) (discussed further below). Synthesis of indole-3-carboxy aspartic acid was performed as described in Supplementary Figure S1. We performed our preliminary screen on a minimal version of MSgg, a common defined biofilm-forming medium (Branda et al. 2001), using ammonium chloride as a nitrogen source instead of glutamate (e.g., $\mathrm{MSg}-\mathrm{NH}_{4} \mathrm{Cl}$ ) to further sensitize the cells to indoles (Bucher et al. 2016). This medium supports the formation of complex colonies that depend on EPS ((Bloom-Ackermann et al. 2016) (Supplementary Figure S2) and the formation of robust floating biofilms (pellicles) (Bucher et al. 2016).

We tested each of these indole derivatives at three concentrations (Fig. 1C). None of the compounds tested had an effect at $50 \mu \mathrm{M}$; three compounds appeared to inhibit pellicle formation at $100 \mu \mathrm{M}$ and more significantly at $500 \mu \mathrm{M}$. To determine whether indole phytohormones can control the biomass of associate beneficial communities, we quantified the floating biomass (discussed below). While quantification of the floating biomass does not provide an accurate estimate of the overall structure of the biofilm, it is considered a robust measurement of the potential treatments (Beauregard et al. 2013). IAOx and IAA significantly reduced biofilm biomass at both $100 \mu \mathrm{M}$ (Supplementary Fig. S3) and $500 \mu \mathrm{M}$ (Fig. 1D) concentrations, and the effect of I3CA-Me was statistically significant at $500 \mu \mathrm{M}$ (Fig. 1D). This preliminary investigation of structureactivity relation (SAR) (Fig. 1A, C, and D) revealed that inhibition of pellicle formation was highly specific. The presence of a methyl moiety in I3CA-Me was sufficient to significantly enhance the biofilm-inhibiting properties of I3CA, suggesting the existence of a bacterial recognition mechanism of the side group in these host metabolites. Moreover, all three compounds disrupted the architecture of structured colonies on a solid biofilm medium (Fig. 1E). Colony biofilm structure was more sensitive to root-derived indoles than were floating biofilms, as the colony morphology was affected by several additional rootderived indoles, such as I3CA (Supplementary Fig. S4).

Importantly, the biofilm-inhibitory concentrations of IAOx $(100 \mu \mathrm{M})($ Supplementary Fig. S5) and I3CA-Me $(500 \mu \mathrm{M})$ (Fig. $1 F)$ had little effect on planktonic cell growth. IAA slightly induced planktonic growth at $100 \mu \mathrm{M}$ but had no effect on planktonic growth at $500 \mu \mathrm{M}$ (Fig. 1F). In contrast, $500 \mu \mathrm{M} \mathrm{I} 3 \mathrm{COH}$ significantly impaired planktonic growth (Fig. 1F) but had no effect on biofilm formation (Fig. 1C and D). These results indicate that IAOx, IAA, and I3CA-Me have a specific effect on biofilm development without negatively affecting cell growth. We therefore postulate that the plant-derived metabolites can function as regulators to limit floating biofilm formation in root-associated communities. Furthermore, the lack of effect of both tryptophan and indole strongly supports the hypothesis that a root-derived indole structure is essential for the bacterial response.

We continued to evaluate the robustness of the bacterial response to IAOx on a biofilm medium in which an optimal nitrogen source, glutamate, was used (Bloom-Ackermann et al. 2016; Hassanov et al. 2018). Here again, the development of both floating biofilms (Supplementary Fig. S6) and biofilm colonies (Fig. 2A) was disrupted by IAOx and, to a lesser extent, by I3CAMe and IAA, although the minimal inhibitory concentration for biofilm formation was fivefold higher. Again, biofilm inhibitory concentrations of I3CA-Me and IAOx had no significant effect on the rate of planktonic growth and even slightly improved carrying capacity in MSgg (Supplementary Fig. S7), indicating that rootproduced indole derivatives specifically regulate biofilm formation independently of the nitrogen source. As biofilm colonies in this medium develop a highly complex morphology, we could pinpoint the appearance of the defect in the formation of concentric rings and complex ridges to more than 24 but less than $48 \mathrm{~h}$ postinoculation (Supplementary Fig. S8). Neither the plant-produced tryptophan nor the bacteria-produced indole exhibited biofilmregulating properties for $B$. subtilis (Fig. 2A), further supporting the idea that the arrest of biofilm development at a given biomass is a specific response to phytohormones.

Fig. 1. Root-derived indoles specifically inhibit floating biofilm formation. A, A single metabolic pathway contributes to the production of root-derived indoles (Iven et al. 2012). B, Synthesis of indole-3-acetaldoxime (IAOx). Reagents and conditions for a: sulfur trioxide pyridine complex (3 eq), dimethyl sulfoxide (DMSO), triethylamine (7 eq), dry $\mathrm{CH}_{2} \mathrm{Cl}_{2}, 30 \mathrm{~min}, 25^{\circ} \mathrm{C}, \mathrm{pH} 4.5$ to 5 ; for b: hydroxylamine hydrochloride, sodium acetate, ethanol, 18 -h retention time; $24 \%$ overall yield of the reactions (for both stages). C, Floating biofilm formation. Top-down images of floating biofilms grown in 12-well plates under static conditions in liquid biofilm-inducing MSgg medium in which the nitrogen source was replaced by ammonium chloride at an equimolar ratio $\left(\mathrm{MSg}-\mathrm{NH}_{4} \mathrm{Cl}\right)$ for 4 days at $23^{\circ} \mathrm{C}$, with root-derived indoles at indicated concentrations. DMSO added in a vol/vol ratio identical to the $500 \mu \mathrm{M}$ indole derivatives solution served

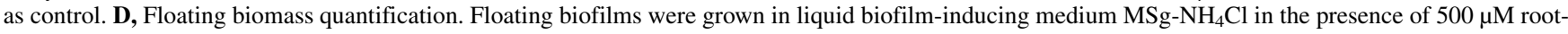
derived indoles, and their mass was determined. Shown is the ratio between biomass of treated biofilms and the untreated controls. The results are an average of three independent experiments, error bars represent standard deviation. An asterisk (*) indicates $P$ value $<0.01$ (determined by two-tailed paired $t$ test) compared with the DMSO control. E, Biofilm colony morphology. Top-down images of biofilm colonies grown on solid biofilm-inducing medium MSg$\mathrm{NH}_{4} \mathrm{Cl}$ for $96 \mathrm{~h}$ at $30^{\circ} \mathrm{C}$ in the absence or presence of $500 \mu \mathrm{M}$ root-derived indoles. An equivalent volume of DMSO served as control. Scale bar $=2 \mathrm{~mm}$. $\mathbf{F}$, Planktonic growth. Cultures were grown in liquid biofilm-inducing medium $\mathrm{MSg}-\mathrm{NH}_{4} \mathrm{Cl}$, in a microplate reader, with shaking at $30^{\circ} \mathrm{C}$. Cultures were supplemented with $500 \mu \mathrm{M}$ root-derived indoles or DMSO as a control and optical density at $600 \mathrm{~nm}$ was monitored. Results are averages of five wells within one experiment, error bars represent standard deviations. A representative of at least three independent experiments is shown. An asterisk (*) indicates $P$ value $<0.01$ (determined by two-tailed paired $t$ test) compared with the DMSO control.
} 
To better evaluate the contribution of the structure to the biofilm inhibiting activity, we tested our battery of root-derived indoles on mutants with subtle biofilm defects (Fig. 2A). In these backgrounds, EPS assembly is partial, and thus, the activity of less-potent root-derived indoles could become evident. We tested a mutant for the ywqC-F operon (encoding also the tyrosine-kinase tkmA) partially impairing colony morphology and synthesis of exopolysaccharides (Mijakovic et al. 2003) and a $\Delta \operatorname{tas} A$ mutant impaired in the synthesis of the matrix proteinaceous component (Branda et al. 2006). In the ywqC-F background, IAOx and I3CA-Me decreased biofilm formation, but other derivatives also had a subtler effect (IAN, I3COH, and IAA). The effect of the derivatives on $\Delta \operatorname{tas} A$ was less pronounced. In both backgrounds, the changes in colony morphology were more evident than in the $\Delta$ eps $H$ mutant completely blocked for exopolysaccharides synthesis (Fig. 2A). Root-derived indoles had a subtle impact on the size of the colony in an $\Delta$ eps $H$ mutant. However, the overall morphology remained largely unaffected.

It was previously shown that plant cell-wall polysaccharides can induce the formation of biofilms in B. subtilis (Beauregard et al. 2013), implying that biofilm formation in the root microenvironment can be highly influenced by the leakage of polysaccharides from the plant. To consider a potential interaction between the effects of root-derived indoles and plant polysaccharides, we repeated the experiment in the same optimal growth media, in which the carbon source glycerol was replaced with the plant polysaccharide xylan (Beauregard et al.
A

Control
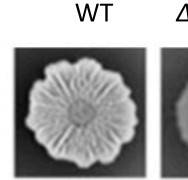

Trp

Indole
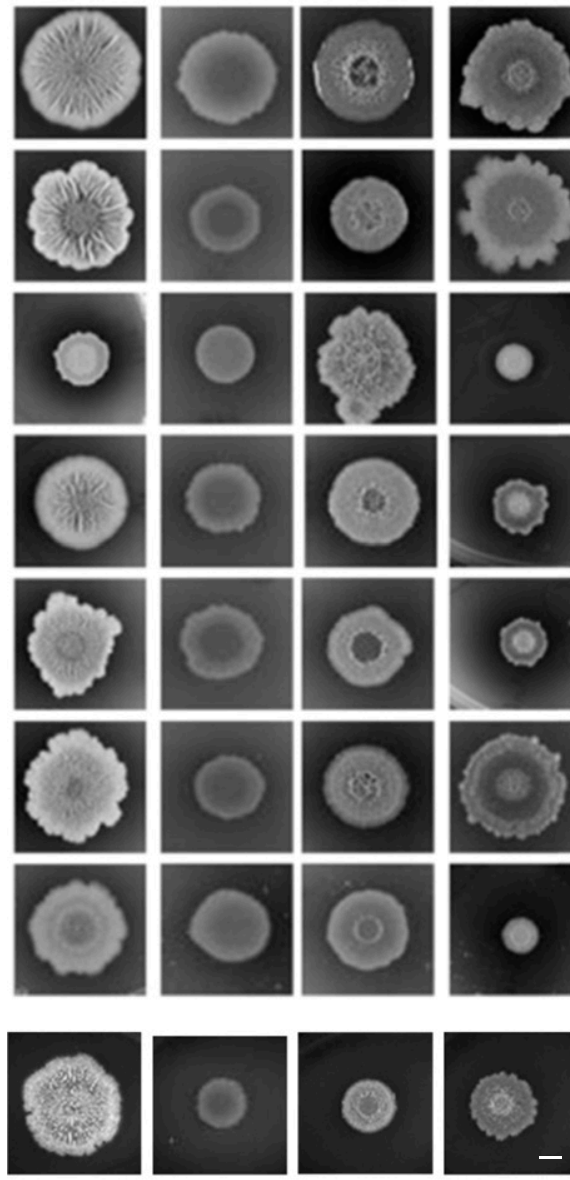

B
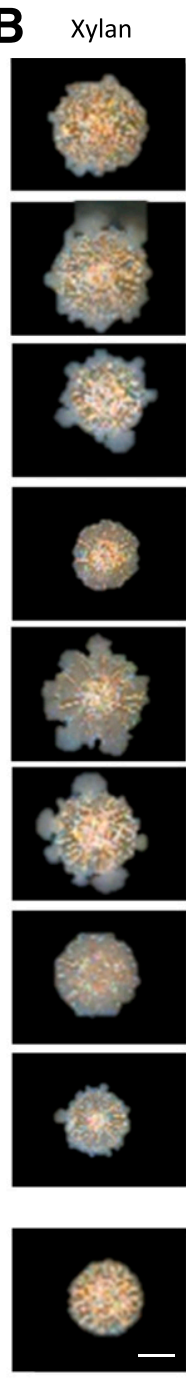

C

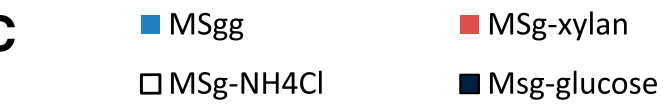

- LBGM

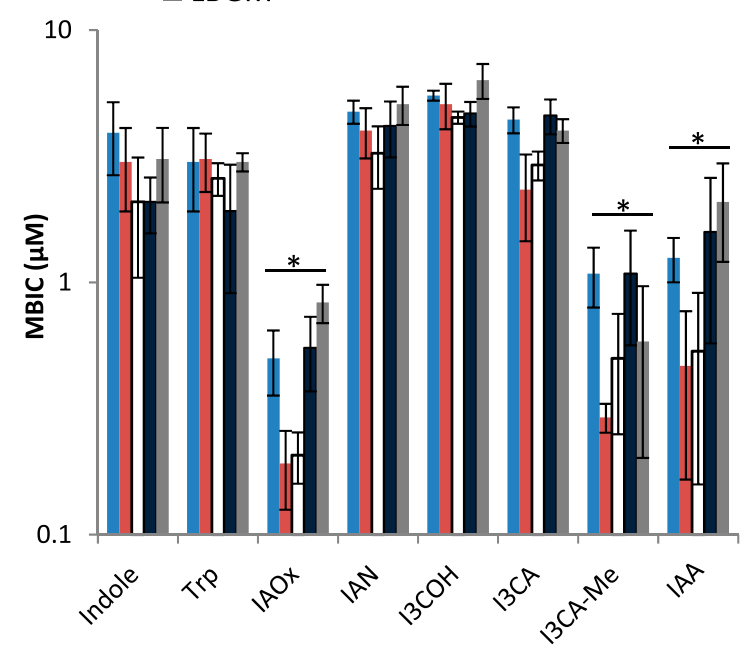

D

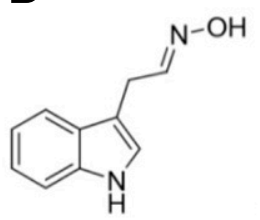

Log P: 1.2 MR: $51\left[\mathrm{~cm}^{3} / \mathrm{mol}\right]$ tPSA: 44.62
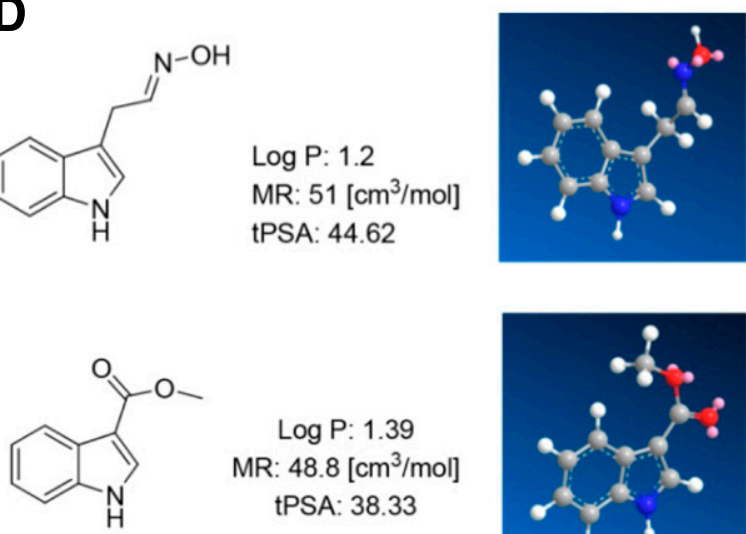

Log P: 1.39 MR: $48.8\left[\mathrm{~cm}^{3} / \mathrm{mol}\right]$ tPSA: 38.33

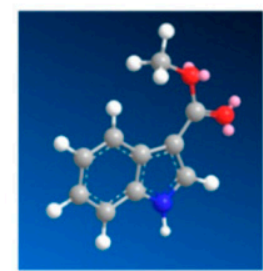

Fig. 2. Side group recognition of indole-3-acetaldoxime (IAOx) and I3CA-Me determines the mode of biofilm inhibition. A, Biofilm colony morphology. Topdown images of biofilm colonies formed by the indicated strains grown under static conditions on solid biofilm-inducing medium MSgg for 3 days at $23^{\circ} \mathrm{C}$, in the absence or presence of $1 \mathrm{mM}$ root-derived indoles. An equivalent volume of dimethyl sulfoxide (DMSO) served as control. The effect of indole-3-acetic acid (IAA) was documented in a separate experiment with an appropriate control, comparable to the included control. Scale bar $=2 \mathrm{~mm}$. B, Biofilm colony morphology in the presence of xylan as a carbon source. Top-down images of biofilm colonies formed by the indicated strains grown under static conditions on solid biofilm-inducing medium MSgg in which glycerol was replaced with $1 \%$ xylan for 3 days at $30^{\circ} \mathrm{C}$, in the absence or presence of $100 \mu \mathrm{M}$ root-derived indoles. An equivalent volume of DMSO served as control. The effect of IAA was documented in a separate experiment with an appropriate control, comparable to the included control. Scale bar $=2 \mathrm{~mm}$. C, Quantification of effects of indole derivatives across media. Floating biofilms were grown on the indicated media for up to 4 days at $23^{\circ} \mathrm{C}$. The minimum biofilm inhibiting concentration (MBIC) was determined as the concentration required for the inhibition of floating biofilm formation compared with a DMSO control for over $12 \mathrm{~h}$ and was consistent with the concentration required to reduce the formation of complex colony morphology. For each growth medium indicated, indoles were tested at the following concentrations: $0.025,0.05,0.1,0.2,0.5$, $0.75,1,2,3,4,5$, and $7 \mathrm{mM}$ in triplicates. An asterisk (*) indicates $P$ value $<0.01$ of the media group treated with the indicated indole compared with the DMSO control. The MBIC values for IAOx differed by a $P$ value of 0.058 between $\mathrm{MSg}-\mathrm{NH}_{4} \mathrm{Cl}$ and MSg-xylan and by 0.0091 between $\mathrm{MSgg}$ and $\mathrm{MSg}$-xylan. $\mathbf{D}$, Physicochemical properties of (E)-2-(1H-indol-3-yl)acetaldehyde oxime and methyl 1H-indole-3-carboxylate. The 3D structures were minimized using Chem3D Pro, using the MM2 force field. Both compounds are estimated to have similar logP values (Log P), molar refractivity values (MR), and polar surface areas (tPSA). 
2013) (Fig. 2B) and, again, observed a significant inhibitory effect of IOAx and I3CA-Me. As media composition appeared to have a role in the response of the biofilm cells to indoles (Figs. 1 and 2A and B), the overall structure-activity relationship was investigated quantitatively across several growth media (Fig. 2C). Overall, out of all tested rich and defined media, the use of xylan as a carbon source was most effective in sensitizing $B$. subtilis biofilms to the different bioactive indoles. In a xylan-containing medium, the effect of IAOx was enhanced significantly, with a fivefold decrease of the biofilm inhibitory concentration to $100 \mu \mathrm{M}$, and the inhibitory concentrations of I3CA-Me and IAA also decreased, though to a lesser extent.

Our investigation suggested that the indole derivatives that consistently inhibit biofilm development have unique physicochemical properties. We calculated the $\log \mathrm{P}$ as a hydrophobicity measurement for each compound. The $\log \mathrm{P}$ value is the partition coefficient (or distribution of concentrations) for a compound between two immiscible phases, commonly between octanol and water (Leo et al. 1971). The active compounds are estimated to have similar $\log \mathrm{P}$ values, molar refractivity values, and polar surface areas. While those are rough estimates, they are indicative of the ability of compounds to pass in the same way through bacterial cell membranes and occupy similar binding pockets (Leo et al. 1971) (Fig. 2D).

I3CA-Me acts independently of quorum-sensing pathways and requires the presence of global biofilm regulators.

Next, we wanted to explore whether I3CA-Me and IAOx act by quorum quenching - as competitive inhibitors of the cellular responses to the known quorum-sensing signals. The addition of excess indole did not restore floating biofilm formation in the presence of I3CA-Me and IAOx (Fig. 3A), strongly indicating that the bacterial response to I3CA-Me and IAOx is independent of the indole sensory pathways. The involvement of AI-2 in cell-to-cell communication is established in B. subtilis (Habe et al. 2001; Lombardia et al. 2006). This pheromone was previously suggested to act in parallel to indole, making indole signaling more robust in the absence of AI2 (Ren et al. 2004). Therefore, we tested whether I3CA-Me and IAOx are capable of competing with DPD (4,5-dihydroxy-2,3pentanedione), the precursor of AI-2. In the wild-type strain, we found that I3CA-Me and IAOx were as effective in the presence of an equivalent molar ratio of DPD as in its absence (Fig. 3A). We considered the option that, in a wild-type strain, high levels of AI-2 are being produced and, thus, the receptors for

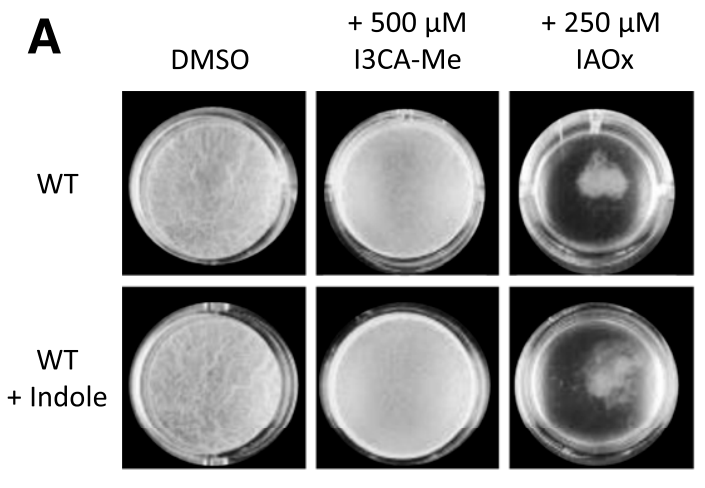

B
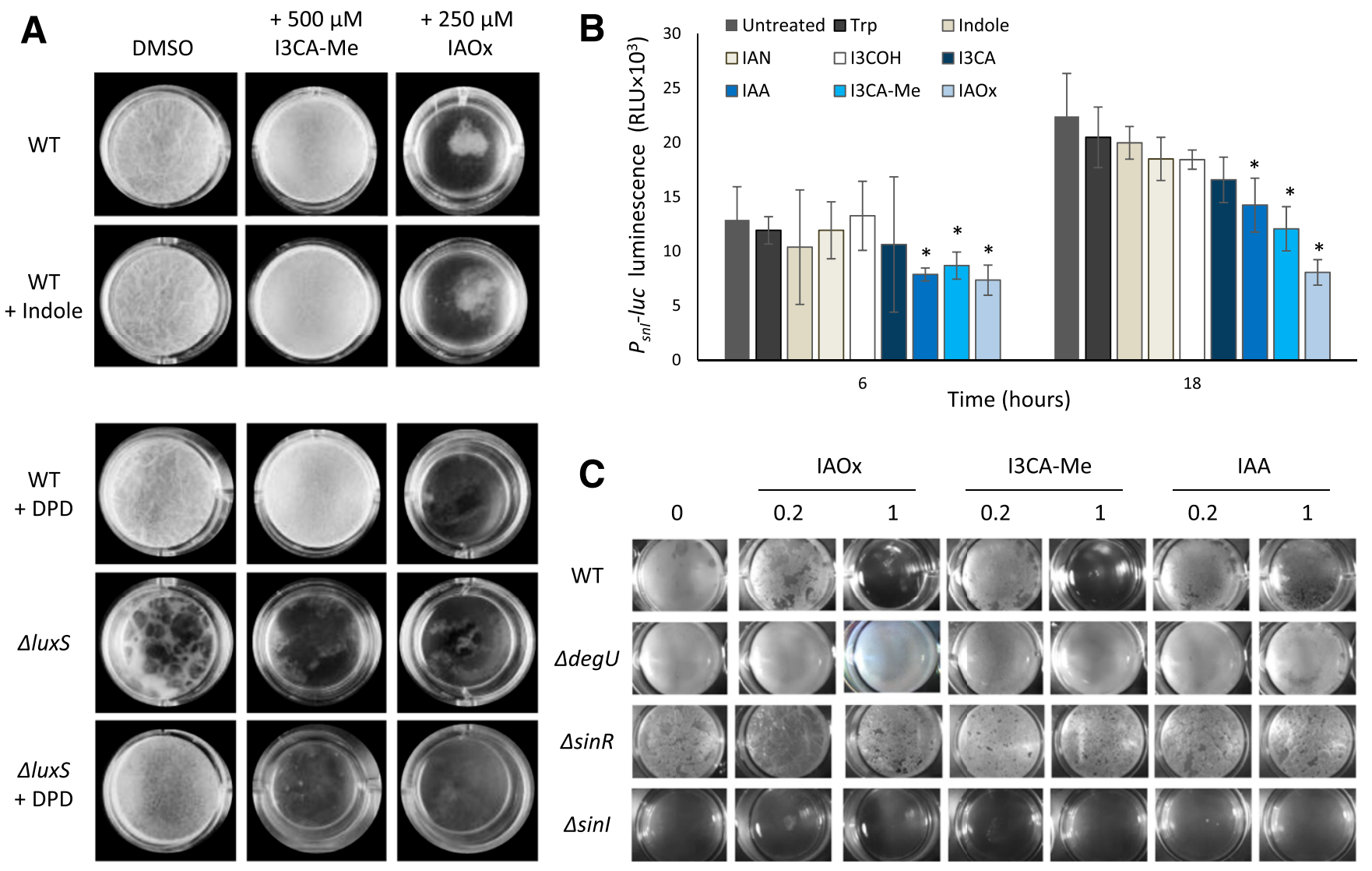

Fig. 3. Indole-3-acetaldoxime (IAOx) and I3Ca-Me do not compete with the endogenic quorum-sensing molecules AI-2 and indole to inhibit floating biofilm formation. A, Floating biofilm formation. Top-down images of floating biofilms grown in 12-well plates under static conditions in liquid biofilm-inducing MSgg medium for 3 days at $23^{\circ} \mathrm{C}$, containing root-derived indoles at biofilm-inhibiting concentrations, as indicated. Dimethyl sulfoxide (DMSO) added in a $\mathrm{vol} / \mathrm{vol} \mathrm{ratio} \mathrm{identical} \mathrm{to} \mathrm{the} 500 \mu \mathrm{M}$ indole derivatives solution served as control. Indole $(500 \mu \mathrm{M})$ or DPD (4,5-dihydroxy-2,3-pentanedione) $(500 \mu \mathrm{M})$ was added as indicated. B, sinI transcription. Data shown represent the level of luciferase (luc) activity in a wild-type strain carrying a luc reporter driven by a sinI promoter. Cells were grown in liquid biofilm-inducing medium MSgg, without or with the indicated indoles $(500 \mu \mathrm{M})$, in a microplate reader with shaking at $30^{\circ} \mathrm{C}$. The values represent normalized units of luminescence (luminescence divided by optical density at $600 \mathrm{~nm}$ ). Results are averages of four wells within one experiment, error bars represent standard deviations. A representative of three independent experiments is shown. An asterisk $(*)$ indicates $P$ value $<0.01$ (determined by two-tailed paired $t$ test) compared with DMSO control. C, The effect of biofilm-deficient mutants on inhibition of floating biofilm formation by root-derived indoles. Top-down images of floating biofilms of wild-type and mutant strains (as indicated) grown in 12-well plates under static conditions in liquid biofilm-inducing $\mathrm{MSg}-\mathrm{NH}_{4} \mathrm{Cl}$ for 4 days at $23^{\circ} \mathrm{C}$, in the absence or presence of indole-3-acetic acid (IAA), IAOx, and I3CA-Me (concentrations are provided in millimoles). DMSO added in a vol/vol ratio identical to the $0.5 \mathrm{mM}$ indole derivatives solution served as control. 
root-derived indoles may be saturated. We repeated the experiment using the luxS mutant incapable of producing AI-2 (DuanisAssaf et al. 2016). The striking effect of luxS deletion on biofilm development suggests a central role for its product in Bacillus biofilms. The addition of high concentrations of DPD (Fig. 3A) but not indole (data not shown) restored biofilm formation partially, confirming the phenotype is due to the loss of a signal molecule reminiscent of AI-2. In this background, similarly to the wild-type parental strain, addition of DPD did not restore biofilm formation in the presence of either I3CA-Me or IAOx (Fig. 3A). Thus, we surmise that the root-derived indoles do not act by quenching the AI-2 or indole pathways.

In $B$. subtilis, the master regulator SinR represses the expression of the ectomycorrhizal (ECM) production operons epsA-O and tapA-sipW-tasA directly, while the transcription factor Spo0A-P induces SinI. SinI acts by protein-protein interactions to neutralize SinR (Chai et al. 2009, 2010a and b; Chu et al. 2008; Cozy et al. 2012; Kobayashi 2008), and thus, in its absence, biofilm formation is blocked (Chai et al. 2008). To elucidate the mechanism that enables biofilm inhibition by I3CA-Me and IAOx, we examined the effect of those compounds on SinI expression. First, we monitored the transcription driven by $\sin I$ promoter-a direct readout for the activity of the genetic pathway resulting in matrix production and biofilm formation. We found that biofilm inhibiting concentration of IAOx, I3CA-Me, and IAA (Fig. 3B) repressed the transcription of sinI. To find the regulator responsible for the inhibition of ECM expression by root-derived indole derivatives, we tested the response of various mutants in the regulatory pathways (Vlamakis et al. 2013) to indole derivatives. We found that mutants lacking DegU and SinR are mostly resistant to the activity of IAA, I3CA-Me, and IAOx, even in millimolar concentrations (Fig. 3C). Both the floating biofilms (Fig. 3C) and the biofilm colonies (Supplementary Fig. S9; data not shown) were resistant to all three bioactive indoles. No floating biofilm were formed under our conditions in a sinI mutant (Fig. 3C), in the presence or absence of IAA, I3CA-Me, and IAOx. Together, these results suggest that root-derived indoles act as regulatory signals downregulating matrix transcription, requiring the DegS/DegU two component system as well as the Spo0A pathway. This hypothesis is consistent with a recent finding that DegU can act to increase Spo0A-P levels and that the two sensory pathways converge (Marlow et al. 2014).

\section{Mapping the influences of bacteria-plant interactions on production of indoles.}

To test the outcomes of exposing the bacteria to naturally produced indoles, we extracted indoles from 'Micro Tom', a dwarf cultivar of tomato (Solanum lycopersicum L.). As expected, an inhibitory signal was observed upon exposure of bacterial cells to tomato plant extract (Fig. 4A) and pellicle development was impaired.

To confirm that root-derived metabolites can limit the development of $B$. subtilis biofilms in planta, we then tested the development of biofilms in the presence of tomato plants on biofilm-inducing media. Biofilms grown with tomato plants were significantly thinner (Fig. 4B). Root-derived indoles prevent de-repression of SinR by inhibiting SinI (Fig. 3B and C), and, consistently, the effects of the plant roots were dependent on the presence of the regulatory cascade for biofilm formation; tomato roots had no effect on biofilm development in the absence of the $\sin R$ (Fig. 4B). This pathway also determined the specificity of colonization, as the wild-type bacterial cells colonized the root area specifically, while $\Delta \sin R$ mutants were also capable of colonizing leaves (Fig. 4C).

We then examined the levels of various indole derivatives in B. subtilis, the model plants tomato Micro Tom, and Eruca sativa, belonging to the Baricasse family. IAOx was not detected in any of the samples, potentially as it is prone to interconversions (Iven et al. 2012). Our inability to detect IAOx in our system still leaves an intriguing possibility that it is produced and accumulated under pathological conditions. I3CA-Me was secreted by tomato plants (Fig. 4D and E), E. sativa secreted detectable levels of IAN (Supplementary Fig. S10), IAA and I3CA were primarily produced by B. subtilis biofilms (Fig. 4F and G), and $\mathrm{I} 3 \mathrm{COH}$ was produced by both plants and bacteria. The symbiotic interaction significantly enhanced the production of I3CA-Me (Fig. 4D and E), IAA (Fig. 4F and G), and I3CA. In the natural environment of the rhizosphere, complex interspecies interactions lead to a production of a wide array of indole derivatives, which might act in synergy. And indeed, when added together, IAA and I3CA-Me were active at lower concentration than when added alone - their mixture is active at $50 \mu \mathrm{M}$ (Fig. 5A; Supplementary Fig. S11), a concentration compatible with the physiological range within the rhizosphere (Badenoch-Jones et al. 1982; Iven et al. 2012).

$\Delta \sin R$ mutant strain is resistant to the inhibitory effects of IAOx and I3CA-Me. It was used to assess the physiological impact of impairing this sensing pathway and allowing unlimited growth of a beneficial biofilm. $\Delta \sin R$ biofilms reduced the length of the central root, the average number of lateral roots, and the number of leaves by the equivalent of $50 \%$ (as compared with plants grown with the wild-type strain) (Fig. 5B). In contrast, the wild type, similarly to a $\Delta \operatorname{deg} U$ mutant, promoted the growth of coinoculated tomato plants.

As we found that plant polysaccharides (Fig. 2C) act specifically to enhance the sensitivity of $B$. subtilis to IAOx and other root-derived indoles, we suspected that the plant host might inhibit biofilm development as a defense against consumption of plant polysaccharides by the associated bacteria. We therefore assessed whether plant polysaccharides can serve as a main carbon source for $B$. subtilis biofilms. In the absence of a preferred carbon source, B. subtilis can use glutamate as both a carbon and nitrogen source (Gunka and Commichau 2012). In a glutamate-based medium lacking a carbon source, cells grew very poorly and only a thin film was evident (Fig. 5C). A thicker film was noticeable in the presence of plant cellwall polysaccharides xylan and xyloglucan. On solid media, xylan increased the diameter of the colony and enhanced the wrinkling of the biofilms, while xyloglucan had a more subtle effect on colony morphology (Fig. 5C). To test whether plant polysaccharides induce biofilms by promoting growth, we evaluated the effects on bacterial planktonic growth and compared them with the effects on biofilm formation. Xylan induced bacterial growth (Supplementary Fig. S12). The effect was clearly metabolic, as polysaccharide subunits (e.g., glucose and xylose) promoted floating biofilm formation even when provided as monomers (Supplementary Fig. S13), significantly accelerated the logarithmic growth, and greatly increased the maximal cell density obtained (Supplementary Fig. S14). Note that, according to a previous report, in the presence of glycerol, plant polysaccharides can also act as a signal and cannot be fully complemented by their cognate subunits (Beauregard et al. 2013). These results indicate that xylan and xyloglucan can act as efficient primary carbon sources, and this property may explain the need for a plant-derived inhibitory mechanism to control the development of the associated community.

The deleterious effect of $\Delta \sin R$ mutant on plants (Fig. 5B) cannot be explained entirely by overgrowth of the bacteria, as $\Delta \sin R$ has a decreased planktonic growth rate (Lopez et al. 2009) but increased overall biomass due to enhanced EPS production. $\Delta \sin R$ mutants express the EPS operons constitutively, due to the lack of the antirepressor SinI. Therefore, we hypothesized that the production of the EPS is related to the 
harmful effects of nonregulated biofilms on the plant host. The extracellular matrix polymers of B. subtilis biofilms treated with xylan had an increased density per floating biofilm, as judged by the dry weight (Fig. 5D). Furthermore, gas chromatography-mass spectrometry (GC-MS) analysis of the EPS fractions of floating biofilms treated with xylan revealed a peak corresponding to xylose (Fig. 5E and F), suggesting that bacteria were able to break down xylan into xylose subunits and incorporate them into their EPS. This is, to the best of our knowledge, a first direct evidence that a plant polysaccharide can, indeed, be broken down and recycled to the EPSs, changing their chemical properties. Interestingly, biofilms grown on xylan were sensitized to root-derived indoles (Fig. 2C), consistent with the idea of an ecological need to balance the bacterial use of plant polysaccharides as metabolites and building blocks by root-derived indoles.

\section{DISCUSSION}

Bacterial biofilms can confer beneficial effects to other organisms such as plants. Specifically, beneficial biofilms formed by Bacillus spp. on plant roots play an important role in maintaining the optimal rhizosphere environment that supports plant growth and fitness. In addition, they may confer immunity against a wide range of foliar diseases by activating plant defenses (Bever et al. 2012; Chen et al. 2013). This protection is proposed to be mediated, in part, by the formation of biofilms (Bais et al. 2004; Raaijmakers et al. 2010). A recent work highlighted indole-derived metabolites synthesis as an important pathway in recruiting $B$. subtilis to Arabidopsis roots (Huang et al. 2015). Here, we show that indoles control the development of biofilms formed by the beneficial bacterium B. subtilis. SAR analysis (Fig. 1) revealed that IAOx and I3CA-Me play a cardinal role in limiting biofilm growth. In contrast with IAOx and I3CA-Me, indole, a secondary metabolite produced by both plants and bacteria (Tomberlin et al. 2017), had no effect on the development of $B$. subtilis biofilms. As this molecule was implicated to be involved in interspecies communication (Lee et al. 2007, 2015; Molina-Santiago et al. 2014), it is feasible that indole regulates other traits of $B$. subtilis.

Accumulation of specific indoles in the root-microenvironments can limit biofilm thickness while overcoming the quorumsensing signal AI-2 (Fig. 3). Importantly, B. subtilis biofilms degrade various plant polysaccharides (Fig. 5), using them both

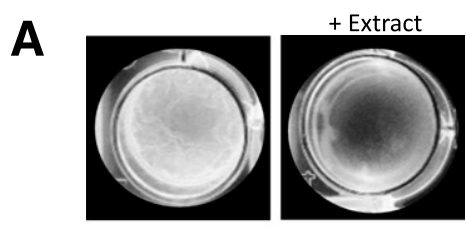

B

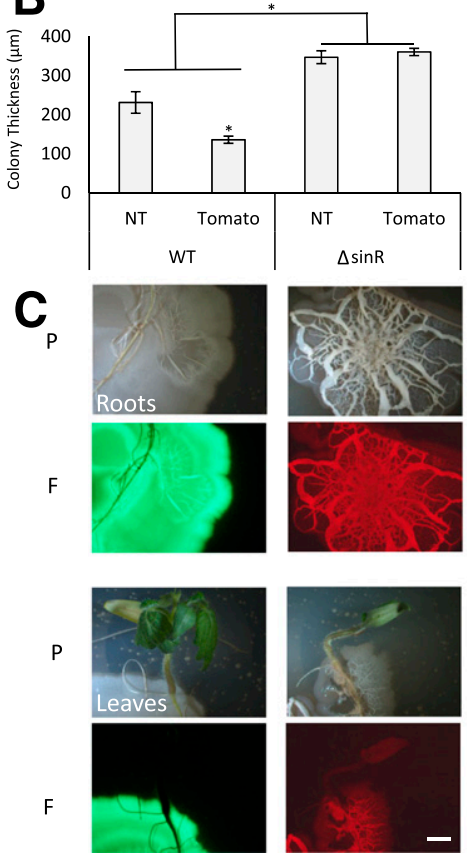

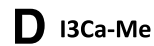
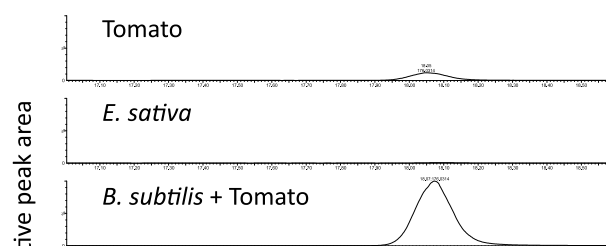

$\frac{\pi}{\mathscr{T}}$

B. subtilis + E. sativa

B. subtilis

Retention time (min)

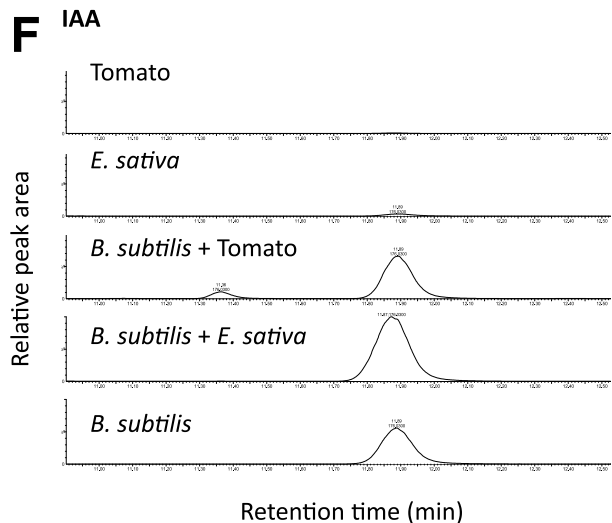

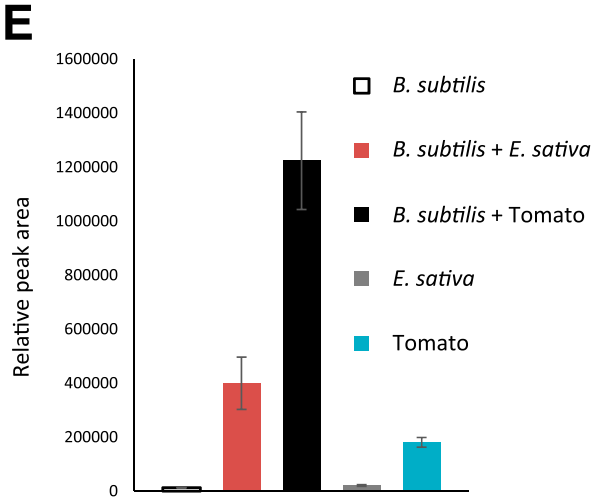

G

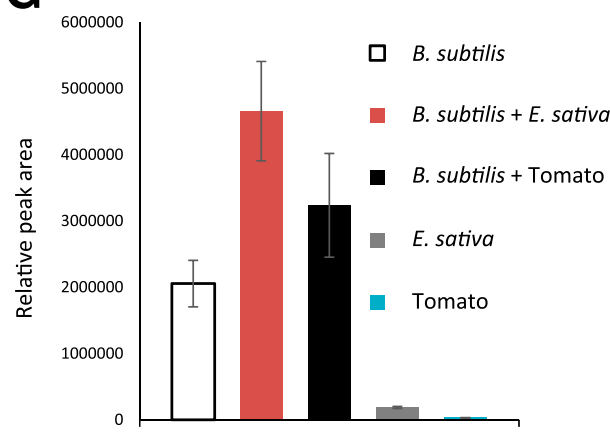

Fig. 4. Indole derivatives accumulation during plant-bacteria interactions. A, Floating biofilm formation. Top-down images of floating biofilms grown in 12well plates under static conditions in liquid biofilm-inducing MSgg, either without or with $10 \mu \mathrm{l}$ of a tomato extract, and purified on C-18 cartridge. Tomato plants were extracted and the solvent was evaporated as previously described (Rosenberg et al. 2016) and was dissolved in double-distilled $\mathrm{H}_{2} \mathrm{O}$. B and $\mathbf{C}$, Rootassociated biofilm formation. A wild-type Bacillus subtilis strain labeled with green fluorescent protein and a $\Delta$ sinR mutant strain labeled with mKate 2 were coinoculated with tomato plants on solid biofilm-inducing medium MSgg and were grown 4 days. B, Quantification of biofilm thickness. Results are averages of three independent experiments $(n=5)$, error bars represent standard deviations. An asterisk $(*)$ indicates $P$ value $<0.01$ (determined by two-tailed paired $t$ test) compared with control (NT = not treated). C, Colonization patterns of the tomato plant by B. subtilis biofilms. Phase (P) and fluorescent (F) channels for roots and leaves are provided. A representative of four independent experiments $(n=5)$ is shown. Scale bar $=1.5 \mathrm{~mm}$. D to $\mathbf{G}$, Liquid chromatography-tandem mass spectrometry (LC-MS/MS) analysis. D, Representative chromatogram of LC-MS/MS analysis of I3CA-Me in Eruca sativa, tomato, and B. subtilis extracts obtained in isolation and during symbiotic interactions. E, Quantification of three independent repeats performed in at-least duplicates of LC-MS/MS of I3CA-Me in E. sativa, tomato, and B. subtilis extracts obtained in isolation and during symbiotic interactions. F, Representative chromatogram for LCMS/MS analysis of indole-3-acetic acid (IAA) in E. sativa, tomato, and B. subtilis extracts obtained in isolation and during symbiotic interactions. G, Quantification of three independent repeats performed in at-least duplicates of LC-MS/MS of IAA in E. sativa, tomato, and B. subtilis extracts obtained in isolation and during symbiotic interactions. 
as a carbon source and as a building block for EPS synthesis. Therefore, it is highly feasible that the limitation of the biofilm biomass by these phytohormones (Fig. 1) serves to prevent deleterious effects of overgrowth of beneficial biofilms. Accordingly, our results indicated that root-derived indoles inhibited the activation of EPS genes by acting upstream to the antirepressor SinI (Fig. 3B and C). SinI is central to the transcriptional activation of the EPS operons, as it controls the activity of the master regulator SinR, a repressor of the eps and
tapA-sipW-tasA operons (Branda et al. 2001; Chai et al. 2010b; Kearns et al. 2005). Indeed, in the absence of SinR, EPS components are expressed constantly, and the increased biomass of the biofilm was deleterious to the plant (Fig. 5B). The two-component DegS-DegU system is an additional pathway that regulates the expression of the EPS operons (Mielich-Süss and Lopez 2015). This two-component system was also essential for sensing root-derived indoles and may act upstream to SinI (Marlow et al. 2014). Interestingly, our results indicate
A

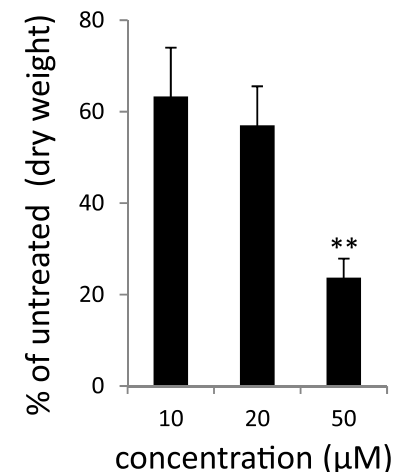

C
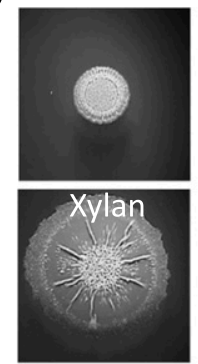

Xyloglucan
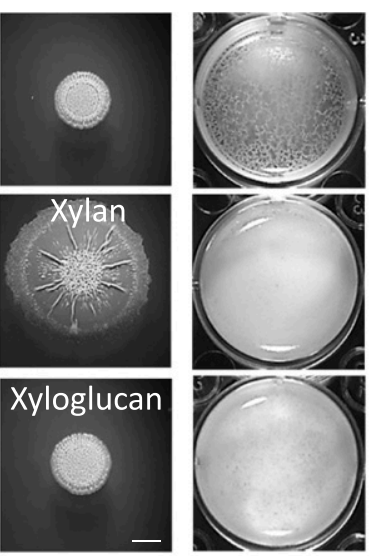

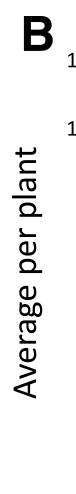

D

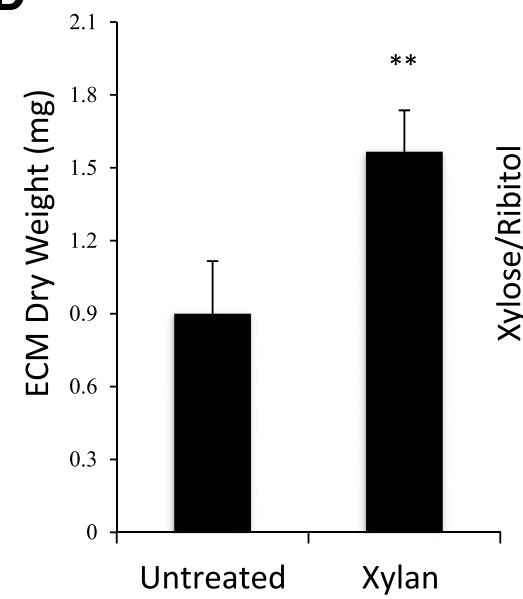

E

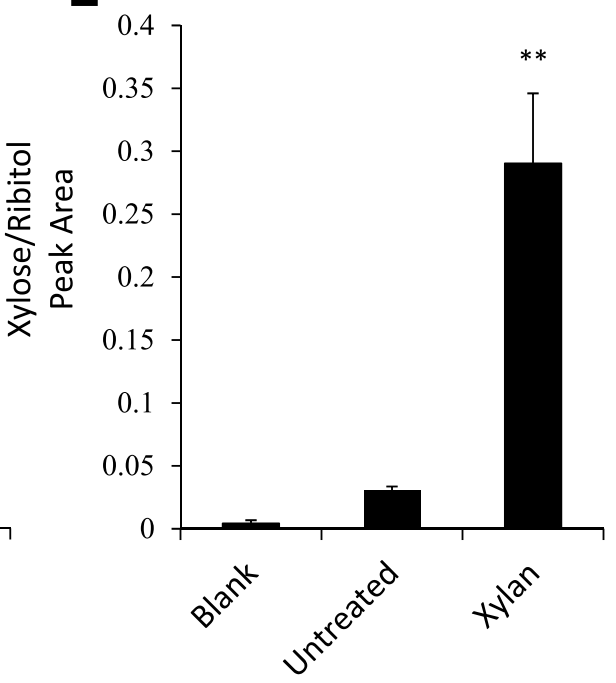

$\mathbf{F}$

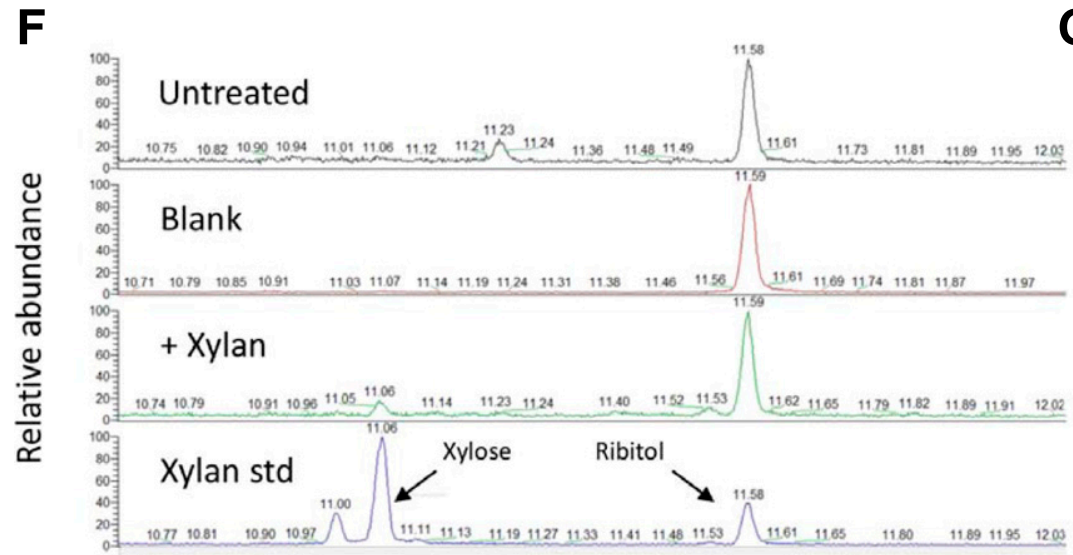

$\mathbf{G}$



Retention time (min) 
that symbiosis is achieved via a complex interaction with the plant host (Fig. 5G). Plant polysaccharides can act as favorable primary carbon sources when added as the main carbon source into the growth medium, as they induced biofilm formation of $B$. subtilis in liquid and on solid biofilm medium. On the other hand, plant polysaccharides also increase the potency of root-derived indoles as inhibitors of biofilm formation. These results may imply that growth in the rhizosphere in carbon limited soils is largely affected by the capacity of the biofilm cells to utilize plant polysaccharides. Furthermore, at least one plant polysaccharide can be degraded and incorporated into the extracellular matrix polymers (Fig. 5E and F). Biofilms growing without restriction, such as biofilms formed by a $\Delta \sin R$ mutant lacking the repressing machinery for the matrix genes, become deleterious to the plant host. In order to remain beneficial, the beneficial communities have to feed from the host without exceeding its carrying capacity. Overall, our work presents novel insights into the complex mechanisms that evolved to sustain beneficial interactions.

\section{MATERIALS AND METHODS}

\section{Synthetic procedures.}

General. Unless otherwise stated, all reactions were performed under an inert atmosphere with dry reagents and solvents. All chemical reagents were purchased from Aldrich, Acros, and Chem Impex Inc. and were used without further purification. Thin-layer chromatography was performed using silica gel 60 with F254 indicator on glass plates (Merck). Flash chromatography was performed using Merck 40- to $63-\mu \mathrm{m}$ silica gel. Solvent ratios for the purification of compounds by flash chromatography are reported as percent volume (vol/vol). Nuclear magnetic resonance (NMR) spectra were recorded using a Bruker Avance DPX $300(300 \mathrm{MHz})$ or Bruker Avance $\mathrm{DMX}_{400}(400 \mathrm{MHz})$ spectrometer. Spectra were calibrated on residual solvent signal.

Synthesis of indole-3-acetaldoxim.

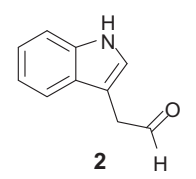

Compound 2. Sulfur trioxide pyridine complex (2.2 g, $13.8 \mathrm{mmol})$ in dimethyl sulfoxide (DMSO) $(3.8 \mathrm{ml})$ and dry dichloromethane (DCM) $(6 \mathrm{ml})$ were added at room temperature under nitrogen to a solution of commercially available indole-3-ethanol (740 $\mathrm{mg}, 4.6 \mathrm{mmol})$, DMSO $(3.8 \mathrm{ml})$, and triethylamine $(4.4 \mathrm{ml}, 31.5 \mathrm{mmol})$ in dry DCM $(10 \mathrm{ml})$. The mixture was stirred at room temperature for $1 \mathrm{~h}$ and was monitored by thin layer chromatography (TLC). The reaction mixture was then acidified to $\mathrm{pH} 4.5$ to 5 , was dissolved in water, and was extracted a few times with DCM and EtOAc (ethanol, n-butanol, and ethyl acetate). The combined organic phases were dried with $\mathrm{Na}_{2} \mathrm{SO}_{4}$, filtered and concentrated in vacuo to afford crude indole-3-acetaldehyde (compound 2).



Compound 3. Hydroxyl amine hydrochloride $(638 \mathrm{mg}$, $9.2 \mathrm{mmol}$ ) and sodium acetate $(754 \mathrm{mg}, 9.2 \mathrm{mmol})$ were added into a solution of the crude indole-3-acetaldehyde in ethanol $(30 \mathrm{ml})$ at room temperature. After stirring overnight, the reaction mixture was concentrated in vacuo, was solubilized with DCM, and was chromatographed on silica gel, using dry loading eluted with a gradient mixture of 20 to 30\% EtOAc: hexane to afford indole-3-acetaldoxime (compound 3) $(77 \mathrm{mg}$, mixture of cis/transform, $24 \%$ overall yield).

${ }^{1} \mathrm{H}$ NMR (400 MHz, CD $\left.\mathrm{OD}\right): \delta$ ppm $7.49(\mathrm{~d}, J=7.88 \mathrm{~Hz}$, $1 \mathrm{H}), 7.32(\mathrm{~d}, J=8.16 \mathrm{~Hz}, 1 \mathrm{H}), 7.02$ to $7.06(\mathrm{~m}, 2 \mathrm{H}), 6.94(\mathrm{t}, J=$ $7.45 \mathrm{~Hz}, 1 \mathrm{H}), 6.78(\mathrm{t}, J=5.31 \mathrm{~Hz}, 1 \mathrm{H}), 3.79(\mathrm{~d}, J=5.30$ $\mathrm{Hz}, 1 \mathrm{H})$.

${ }^{13} \mathrm{C}$ NMR (400 MHz, $\left.\mathrm{CD}_{3} \mathrm{OD}\right): \delta 152.07,138.26,128.67$, $123.72,122.55,119.79,119.24,112.32,111.12,22.35$.

Synthesis of indole-3-carboxy aspartic acid.

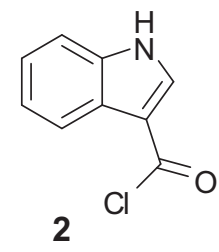

Compound 2. Oxalyl chloride $(60 \mu \mathrm{l}, 0.69 \mathrm{mmol})$ and DMF (one drop) were added to a stirred solution of indole-3carboxylic acid $(100 \mathrm{mg}, 0.6 \mathrm{mmol})$ in DCM $(3 \mathrm{ml})$, with further stirring at ambient temperature for about $2 \mathrm{~h}$. The reaction mixture was concentrated in vacuo to give the desired 1H-indole-3-carbonyl chloride (compound 2).

Fig. 5. Deleterious effects of overgrown biofilm could be due to recycling of plant polysaccharides. A, Floating biomass quantification. Floating biofilms were grown in liquid biofilm-inducing medium $\mathrm{MSg}-\mathrm{NH}_{4} \mathrm{Cl}$ in the presence of indole-3-acetic acid and I3CA-Me mixture at the indicated concentrations and their mass was determined. Shown is the ratio between biomass of treated biofilms and the untreated controls (dimethyl sulfoxide [DMSO] added in a vol/vol ratio identical to the mixture solution served). The results are an average of three independent experiments, error bars represent standard deviation. Two asterisks (**) indicate $P$ value $<0.005$ (determined by two-tailed paired $t$ test) compared with the DMSO control. B, Plant characterization. Evaluation of tomato plants grown in the presence of the different Bacillus subtilis strains for 7 days. The results are an average of three independent experiments $(n=10)$, error bars represent standard deviation. One asterisk (*) indicates $P$ value $<0.01$ and two asterisks $(* *) P$ value $<0.005$ (determined by two-tailed paired $t$ test) compared with untreated control (NT). C, Biofilm formation in the presence of plant polysaccharides. Floating biofilms (in 12-well plates in liquid medium) and biofilm colonies (on solid medium) were grown in biofilm-inducing medium $\mathrm{MSgg}$ at $30^{\circ} \mathrm{C}$, either untreated or supplemented with xylan $(0.7 \%)$ and xyloglucan $(0.7 \%$ for floating biofilm assay, $0.1 \%$ for biofilm colony assay). The results shown are of a representative experiment out of at least three repeats. Scale bar $=2 \mathrm{~mm}$. D, Ectomycorrhizal $(\mathrm{ECM})$ quantification. Dry weight of an extracted ECM of floating biofilm grown either with or without xylan, grown as in A. Two asterisks (**) indicate $P$ value $<0.005$ compared with untreated control. E and $\mathbf{F}$, Gas chromatography-mass spectrometry GC-MS analysis. Shown are quantification (E) and representative total ion chromatograms for GC-MS analysis (F) of exopolysaccharides extracted from the ECM of floating biofilms either with or without xylan, grown as in A. Two asterisks (**) indicate $P$ value $<0.005$ compared with untreated control. G, A proposed mechanism of regulating the biomass of beneficial biofilms by their plant host. B. subtilis biofilms are inhibited by specific indole derivatives (e.g., indole-3acetaldoxime), which act as signals regulating bacterial growth, and are more potent when the plant polysaccharides serve as a carbon source for the bacteria. 


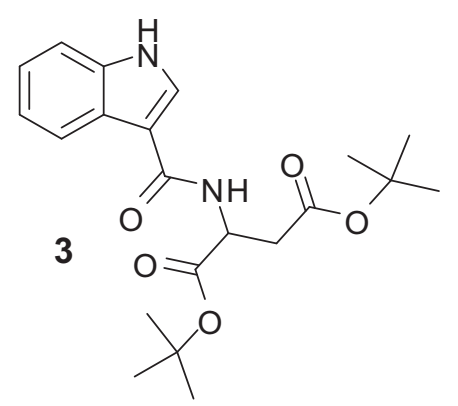

Compound 3. 1H-indole-3-carbonyl chloride (283 mg, $1.56 \mathrm{mmol})$ was dissolved in a mixture of DCM $(3 \mathrm{ml})$ and tetrahydrofuran (THF) (1 ml) and, then, was added dropwise to a stirred solution of $\mathrm{H}-\mathrm{ASP}(\mathrm{OtBu})_{2} \bullet \mathrm{HCl}(444 \mathrm{mg}$, $1.576 \mathrm{mmol})$, trimethylamine $(218 \mu \mathrm{l}, 159.2 \mathrm{mg}, 1.57 \mathrm{mmol})$, and DCM $(3 \mathrm{ml})$. The reaction was stirred at room temperature overnight. Next, the mixture was washed with brine $(5 \mathrm{ml})$ and $10 \% \mathrm{NaHCO}_{3}(5 \mathrm{ml})$. The organic layer was dried over $\mathrm{Na}_{2} \mathrm{SO}_{4}$, was filtered, and was concentrated in vacuo. Further purification was done by flash chromatography, using a gradient mixture of 0 to $25 \%$ DCM:EtOAc to afford compound 3 at $195 \mathrm{mg}, 80 \%$ yield.

${ }^{1} \mathrm{H}$ NMR $\left(400 \mathrm{MHz}, \mathrm{CDCl}_{3}\right.$ ): $\delta \mathrm{ppm} 8.69(\mathrm{~s}, 1 \mathrm{H}), 8.06$ to 8.09 $(\mathrm{m}, 1 \mathrm{H}), 7.80(\mathrm{~d}, J=2.91 \mathrm{~Hz}, 1 \mathrm{H}), 7.40$ to $7.46(\mathrm{~m}, 1 \mathrm{H}), 7.23$ to $7.29(\mathrm{~m}, 1 \mathrm{H}), 7.06(\mathrm{~d}, J=7.91 \mathrm{~Hz}, 1 \mathrm{H}), 4.98(\mathrm{td}, J=8.23$, $4.27 \mathrm{~Hz}, 1 \mathrm{H}$ ), 2.97 (ddd, $J=43.38,17.11,4.28 \mathrm{~Hz}, 2 \mathrm{H}$ ), 1.50 (s, 9H), 1.44 (s, 9H).

${ }^{13} \mathrm{C}$ NMR $\left(400 \mathrm{MHz}, \mathrm{CDCl}_{3}\right): \delta 170.16,170.35,164.57$, 136.24, 128.19, 124.68, 122.95, 121.80, 120.14, 112.29, 111.75, $82.29,81.47,49.08,37.93,28.06,27.97$.<smiles>O=C(O)CC(NC(=O)c1c[nH]c2ccccc12)C(=O)O</smiles>

Compound 4. Compound 3 (195 mg, $0.50 \mathrm{mmol})$ was dissolved in a solution of $\mathrm{HCl} /$ dioxane $(2 \mathrm{ml}, 4 \mathrm{M})$ in an ice bath and the reaction mixture was allowed to stir overnight. TLC detection indicated that the reaction was completed. The reaction mixture was evaporated under high vacuum several times with diethyl ether to remove dioxane, to give compound 4 in quantitative yield.

${ }^{1} \mathrm{H}$ NMR (400 MHz, CD $\left.3 \mathrm{OD}\right): \delta$ ppm 7.95 (dd, $J=6.22$, $2.10 \mathrm{~Hz}, 1 \mathrm{H}), 7.83(\mathrm{~s}, 1 \mathrm{H}), 7.34(\mathrm{dd}, J=6.41,2.07 \mathrm{~Hz}, 1 \mathrm{H})$, 7.02 to $7.13(\mathrm{~m}, 2 \mathrm{H}), 4.91(\mathrm{t}, J=5.75 \mathrm{~Hz}, 1 \mathrm{H}), 2.90(\mathrm{~d}, J=$ $5.88 \mathrm{~Hz}, 2 \mathrm{H})$.

${ }^{13} \mathrm{C}$ NMR (400 MHz, $\left.\mathrm{CD}_{3} \mathrm{OD}\right): \delta 174.81,174.69,167.91$, $138.19,129.97,123.57,122.21,121.34,112.99,111.44,50.28$, 81.47, 37.39.

\section{Strains and media.}

All strains were derived from NCIB 3610, a natural $B$. subtilis isolate that forms robust biofilms (Branda et al. 2001). Additional laboratory strains like B. subtilis PY79 and Escherichia coli DH5 $\alpha$ were used for cloning purposes. A complete strain list is shown in Supplementary Table S1. Strains and plasmids were constructed using standard methods (Sambrook and Russell 2001). All deletion mutations were generated by long-flanking homology PCR mutagenesis (Wach 1996). DNA was first introduced by transformation into strain PY79 and the deletion was further integrated into NCIB 3610 by transformation. Transformation of B. subtilis PY79 and 3610 by natural competence and double recombination with linearized plasmid or genomic DNA was done as described by Wilson and Bott (1968).

The strains were routinely manipulated in Luria-Bertani (LB) broth (Difco), LBGM medium (Shemesh and Chai 2013), or MSgg medium (5 mM potassium phosphate, $100 \mathrm{mM}$ MOPS [pH 7], $2 \mathrm{mM} \mathrm{MgCl}_{2}, 50 \mu \mathrm{M} \mathrm{MnCl}_{2}, 50 \mu \mathrm{M}$ (pellicle assay) and $125 \mu \mathrm{M}$ (biofilm assay) $\mathrm{FeCl}_{3}, 700 \mu \mathrm{M} \mathrm{CaCl}_{2}, 1 \mu \mathrm{M} \mathrm{ZnCl}_{2}, 2 \mu \mathrm{M}$ thiamine, $0.5 \%$ glycerol, $0.5 \%$ glutamate, $50 \mu \mathrm{g}$ per milliliter, each, of threonine, tryptophan, and phenylalanine).

When indicated, the following manipulations were performed on MSgg: i) replacement of the glutamate with ammonium chloride to the final concentration of $0.5 \%$ (Bloom-Ackermann et al. 2016), ii) replacement of glycerol with glucose $(0.5 \%)$, iii) the replacement of glycerol with xylan $(0.7 \%)$, iv) replacement of glycerol with xyloglucan (0.1 to $0.7 \%)$, or v) replacement of the glycerol with xylose. Solid medium contained $1.5 \%$ Bacto agar (Difco). Note that the iron concentration in the solid MSgg medium was 2.5-fold greater compared with the original recipe (Branda et al. 2001), due to an improvement in the overall morphology development.

\section{Biofilm assays.}

Cells were grown in LB from a single colony isolated over LB plates to a mid-logarithmic phase of growth $\left(4 \mathrm{~h}\right.$ at $37^{\circ} \mathrm{C}$ with shaking). To grow biofilms, $1.5 \mu \mathrm{l}$ of starter culture was inoculated on plates of solid MSgg medium and was dried in a biological hood for $45 \mathrm{~min}$ prior to inoculation. Pellicles were grown in liquid MSgg medium in cell-culture multi-dish plates (Thermo Scientific), as indicated in figure legends. For pellicle assays, cells of a mid-logarithmic culture were washed once in MSgg to remove LB prior to the inoculum and the inoculum ratio was $1: 1,000$ or $1: 500$. We used different temperatures for the biofilm and pellicle assays because we observed the greatest range of colony phenotypes on plates grown at $30^{\circ} \mathrm{C}$ and the greatest range of pellicle phenotypes in cultures grown at $23^{\circ} \mathrm{C}$. Photos of colonies and pellicles were acquired with a Nikon D800 camera or a stereomicroscope (Zeiss), and images were optimized for contrast and brightness using Adobe Photoshop. For all the experiments shown in the manuscript, the biofilm images are of a representative experiment out of at least three independent experiments performed in triplicate.

\section{Growth measurements.}

Cells were grown from a single colony isolated over LB plates to a mid-logarithmic phase of growth $\left(4 \mathrm{~h}\right.$ at $37^{\circ} \mathrm{C}$ with shaking). Cells were diluted 1:100 in 150 $\mu \mathrm{l}$ of liquid MSgg medium of each well of a 96-well microplate (Thermo Scientific). Cells were grown, with agitation, at $30^{\circ} \mathrm{C}$ for 14 to $16 \mathrm{~h}$, in a microplate reader (Synergy 2, BioTek), and the optical density at $600 \mathrm{~nm}$ was measured every $15 \mathrm{~min}$. Cells were either grown in the presence or absence of cell wallinhibiting molecules as indicated in each corresponding figure legend.

\section{EPS extraction.}

Floating biofilms were grown for $48 \mathrm{~h}$ at $30^{\circ} \mathrm{C}$ in $600-\mathrm{ml}$ beakers on top of custom-made nets. The floating biomass was then separated from the growth medium. Exopolysaccharides were extracted as follows. Floating biofilms that had been formed in biofilm-inducing medium were collected, were washed twice in phosphate-buffered saline $(137 \mathrm{mM} \mathrm{NaCl}$, 
$2.7 \mathrm{mM} \mathrm{KCl}, 10 \mathrm{mM} \mathrm{Na}_{2} \mathrm{HPO}_{4}, 1.8 \mathrm{mM} \mathrm{KH}_{2} \mathrm{PO}_{4}$ ), were mildly sonicated, and were then centrifuged to remove the cells. The supernatant was mixed with 5 volumes of ice-cold isopropanol and was incubated overnight at $4^{\circ} \mathrm{C}$. Samples were centrifuged at 7,500 $\times \mathrm{g}$ for $10 \mathrm{~min}$ at $4^{\circ} \mathrm{C}$. Pellets were resuspended in a digestion mix of $0.1 \mathrm{M} \mathrm{MgCl}_{2}, 0.1 \mathrm{mg}$ of DNase per milliliter, and $0.1 \mathrm{mg}$ of RNase per milliliter, were mildly sonicated, and were incubated for $4 \mathrm{~h}$ at $37^{\circ} \mathrm{C}$. Samples were extracted twice with phenol-chloroform. The aquatic fraction was dialyzed for $48 \mathrm{~h}$ with Slide-A-Lyzer dialysis cassettes by Thermo Fisher, 3,500 molecular weight cut-off, against distilled (d) $\mathrm{H}_{2} \mathrm{O}$. Samples were lyophilized. The remaining pellet was weighed and, afterward, dissolved in $500 \mu \mathrm{l}$ of $\mathrm{dH}_{2} \mathrm{O}$.

\section{Plant-bacteria coculturing.}

Solanum lycopersicum L. (tomato 'Micro Tom') and E. sativa seeds were sterilized in sealed desiccator with $5 \mathrm{ml}$ of HCL mixed with $100 \mathrm{ml}$ sodium hypochlorite (Sigma), by an incubation of $3 \mathrm{~h}$. The sterilized seeds were transferred to $60 \mathrm{~mm}$ petri dishes with $25 \mathrm{ml}$ of Nitsch medium (Nitsch $0.217 \%$, sucrose $2 \%$, agar $1 \%$ in $\mathrm{dH}_{2} \mathrm{O}, \mathrm{pH} 5.8$ ). Three tomato seeds or ten $E$. sativa seeds were incubated per a single petri dish. Then tomato and E. sativa were treated differentially as follows. Tomato plants were grown for 7 to 8 days in $23^{\circ} \mathrm{C}$, with cycles of 16-h light periods, followed by a 8 -h dark periods). E. sativa seeds were first stored at $4^{\circ} \mathrm{C}$ in darkness for aestivation for $48 \mathrm{~h}$ and were then moved to grow at $23^{\circ} \mathrm{C}$, in cycles of $10 \mathrm{~h}$ of light and $14 \mathrm{~h}$ of darkness for 18 days. After the growth period, the plants were transferred in a laminar hood into 60-mm petri dishes with MSgg medium, a single tomato plant and two E. sativa plants per plate. The plants were inoculated with $1 \mu \mathrm{l}$ of $B$. subtilis culture, unless indicated otherwise, were grown to a mid-logarithmic stage as described above in the biofilm assay section, and were grown in cycles of $8 \mathrm{~h}$ of a dark, followed by $16 \mathrm{~h}$ of light for 4 days.

\section{Quantification of the thickness of root-associated biofilms.}

Tomato plants were grown with $B$. subtilis biofilms formed by strains carrying a constant-expression fluorescent construct as described above. After 4 days, biofilms were sectioned, as described by us previously (Keren-Paz et al. 2018), and the maximal thickness of the biofilm colony was determined.

\section{Plant extract analysis.}

For extract analysis, samples were then collected, weighed, and ground to a fine powder under liquid nitrogen. Dried samples were stored at $-80^{\circ} \mathrm{C}$ for further analysis. Samples were reconstituted to $50 \mu \mathrm{l}$ with $50: 50 \% \mathrm{ACN} / \mathrm{ddH}_{2} \mathrm{O}$ (double distilled $\mathrm{H}_{2} \mathrm{O}$ ), were vortexed, sonicated, and centrifuged at $13,500 \times g$, and the supernatant was transferred to ultraperformance liquid chromatographic (UPLC) vials for analysis.

Indoles were measured by UPLC-electrospray ionizationtandem mass spectrometry (ESI-MS/MS) equipped with a Waters Acquity UPLC (I class) system with binary pump and Acquity UPLC BEH C18 column $(1.7 \mu \mathrm{m}, 2.1 \times 100 \mathrm{~mm}$, Waters). Compounds were separated at a flow rate of $0.3 \mathrm{ml} / \mathrm{min}$ with a linear gradient elution program with solvent A $(0.1 \%$ formic acid in $5 \%$ acetonitrile- $95 \%$ water $)$ and solvent $\mathrm{B}(0.1 \%$ formic acid in acetonitrile): 0 to $28 \%$ solvent $\mathrm{B}$ ( $22 \mathrm{~min}$ ), 28 to $40 \%$ solvent B (to $22.5 \mathrm{~min}$ ), 40 to $100 \%$ solvent B (to $23 \mathrm{~min}$ ), $100 \%$ solvent B (to $26.5 \mathrm{~min}$ ), and $100 \%$ solvent A (to $28 \mathrm{~min}$ ). The autosampler was cooled to $14^{\circ} \mathrm{C}$ and the column was heated to $35^{\circ} \mathrm{C}$. The MS detector (Waters TQ XS) was equipped with an ESI source used in positive (capillary voltage $2.5 \mathrm{kV}$ ). The measurement was performed in multiple reaction monitoring (MRM) mode. The cone voltages $(\mathrm{V})$ for each compound and collision energies (eV) for each MRM transition were determined by direct injection (Supplementary Table S2). Data were processed with MassLynx software with TargetLynx (version 4.2; Waters).

\section{GC-MS analysis of EPS.}

EPS fractions were hydrolyzed by $2 \mathrm{M}$ triflouroacetic acid and heat hydrolysis as published previously (Ren et al. 2014). The acid was dried using air flow and was then rehydrated with $\mathrm{ddH}_{2} \mathrm{O}$. The internal standard ribitol was added to each sample and the samples were freeze-dried by lyophilization. Derivation and analysis of xylose in the hydrolyzed samples $(n=3)$ was carried out as previously described (Mintz-Oron et al. 2008). Xcalibur version 1.4 (Thermo Finnigan) was used for data analysis. Xylose was identified by comparison of its retention time and mass spectrum to those generated from authentic standard analyzed in the same injection sequence. The response values of xylose resulting from the Xcalibur processing were normalized to the ribitol (adonitol; Sigma Aldrich) internal standard.

\section{LITERATURE CITED}

Allard-Massicotte, R., Tessier, L., Lécuyer, F., Lakshmanan, V., Lucier, J. F., Garneau, D., Caudwell, L., Vlamakis, H., Bais, H. P., and Beauregard, P. B. 2016. Bacillus subtilis early colonization of Arabidopsis thaliana roots involves multiple chemotaxis receptors. MBio 7:7.

Asally, M., Kittisopikul, M., Rué, P., Du, Y., Hu, Z., Çağatay, T., Robinson, A. B., Lu, H., Garcia-Ojalvo, J., and Süel, G. M. 2012. Localized cell death focuses mechanical forces during 3D patterning in a biofilm. Proc. Natl. Acad. Sci. U.S.A. 109:18891-18896.

Badenoch-Jones, J., Summons, R. E., Djordjevic, M. A., Shine, J., Letham, D. S., and Rolfe, B. G. 1982. Mass spectrometric quantification of indole-3-acetic acid in rhizobium culture supernatants: Relation to root hair curling and nodule initiation. Appl. Environ. Microbiol. 44: 275-280.

Bais, H. P., Fall, R., and Vivanco, J. M. 2004. Biocontrol of Bacillus subtilis against infection of Arabidopsis roots by Pseudomonas syringae is facilitated by biofilm formation and surfactin production. Plant Physiol. 134:307-319.

Beauregard, P. B., Chai, Y., Vlamakis, H., Losick, R., and Kolter, R. 2013. Bacillus subtilis biofilm induction by plant polysaccharides. Proc. Natl. Acad. Sci. U.S.A. 110:E1621-E1630.

Bever, J. D., Platt, T. G., and Morton, E. R. 2012. Microbial population and community dynamics on plant roots and their feedbacks on plant communities. Annu. Rev. Microbiol. 66:265-283.

Bloom-Ackermann, Z., Steinberg, N., Rosenberg, G., OppenheimerShaanan, Y., Pollack, D., Ely, S., Storzi, N., Levy, A., and KolodkinGal, I. 2016. Toxin-antitoxin systems eliminate defective cells and preserve symmetry in Bacillus subtilis biofilms. Environ. Microbiol. 18: 5032-5047.

Branda, S. S., Chu, F., Kearns, D. B., Losick, R., and Kolter, R. 2006. A major protein component of the Bacillus subtilis biofilm matrix. Mol. Microbiol. 59:1229-1238.

Branda, S. S., González-Pastor, J. E., Ben-Yehuda, S., Losick, R., and Kolter, R. 2001. Fruiting body formation by Bacillus subtilis. Proc. Natl. Acad. Sci. U.S.A. 98:11621-11626.

Branda, S. S., González-Pastor, J. E., Dervyn, E., Ehrlich, S. D., Losick, R., and Kolter, R. 2004. Genes involved in formation of structured multicellular communities by Bacillus subtilis. J. Bacteriol. 186: 3970-3979.

Branda, S. S., Vik, S., Friedman, L., and Kolter, R. 2005. Biofilms: The matrix revisited. Trends Microbiol. 13:20-26.

Bryers, J. D. 2008. Medical biofilms. Biotechnol. Bioeng. 100:1-18.

Bucher, T., Kartvelishvily, E., and Kolodkin-Gal, I. 2016. Methodologies for studying B. subtilis biofilms as a model for characterizing small molecule biofilm inhibitors. J. Vis. Exp. 116. Published online. doi.org/10.3791/54612

Bucher, T., Oppenheimer-Shaanan, Y., Savidor, A., Bloom-Ackermann, Z., and Kolodkin-Gal, I. 2015. Disturbance of the bacterial cell wall specifically interferes with biofilm formation. Environ. Microbiol. Rep. 7:990-1004.

Cairns, L. S., Marlow, V. L., Bissett, E., Ostrowski, A., and Stanley-Wall, N. R. 2013. A mechanical signal transmitted by the flagellum controls signalling in Bacillus subtilis. Mol. Microbiol. 90:6-21. 
Chai, Y., Chu, F., Kolter, R., and Losick, R. 2008. Bistability and biofilm formation in Bacillus subtilis. Mol. Microbiol. 67:254-263.

Chai, Y., Kolter, R., and Losick, R. 2009. Paralogous antirepressors acting on the master regulator for biofilm formation in Bacillus subtilis. Mol. Microbiol. 74:876-887.

Chai, Y., Kolter, R., and Losick, R. 2010a. Reversal of an epigenetic switch governing cell chaining in Bacillus subtilis by protein instability. Mol. Microbiol. 78:218-229.

Chai, Y., Norman, T., Kolter, R., and Losick, R. 2010b. An epigenetic switch governing daughter cell separation in Bacillus subtilis. Genes Dev. 24:754-765

Chen, Y., Yan, F., Chai, Y., Liu, H., Kolter, R., Losick, R., and Guo, J. H 2013. Biocontrol of tomato wilt disease by Bacillus subtilis isolates from natural environments depends on conserved genes mediating biofilm formation. Environ. Microbiol. 15:848-864.

Chu, F., Kearns, D. B., Branda, S. S., Kolter, R., and Losick, R. 2006. Targets of the master regulator of biofilm formation in Bacillus subtilis. Mol. Microbiol. 59:1216-1228.

Chu, F., Kearns, D. B., McLoon, A., Chai, Y., Kolter, R., and Losick, R. 2008. A novel regulatory protein governing biofilm formation in Bacillus subtilis. Mol. Microbiol. 68:1117-1127.

Cozy, L. M., Phillips, A. M., Calvo, R. A., Bate, A. R., Hsueh, Y. H., Bonneau, R., Eichenberger, P., and Kearns, D. B. 2012. SlrA/SinR/SlrR inhibits motility gene expression upstream of a hypersensitive and hysteretic switch at the level of $\sigma(\mathrm{D})$ in Bacillus subtilis. Mol. Microbiol. 83:1210-1228.

Duanis-Assaf, D., Steinberg, D., Chai, Y., and Shemesh, M. 2016. The LuxS based quorum sensing governs lactose induced biofilm formation by Bacillus subtilis. Front. Microbiol. 6:1517.

Emmert, E. A., and Handelsman, J. 1999. Biocontrol of plant disease: A (gram-) positive perspective. FEMS Microbiol. Lett. 171:1-9.

Grau, R. R., de Oña, P., Kunert, M., Leñini, C., Gallegos-Monterrosa, R., Mhatre, E., Vileta, D., Donato, V., Hölscher, T., Boland, W., Kuipers, O. P., and Kovács, A. T. 2015. A duo of potassium-responsive histidine kinases govern the multicellular destiny of Bacillus subtilis. MBio 6 : e00581-15.

Gunka, K., and Commichau, F. M. 2012. Control of glutamate homeostasis in Bacillus subtilis: A complex interplay between ammonium assimilation, glutamate biosynthesis and degradation. Mol. Microbiol. 85: 213-224.

Habe, H., Ide, K., Yotsumoto, M., Tsuji, H., Hirano, H., Widada, J., Yoshida, T., Nojiri, H., and Omori, T. 2001. Preliminary examinations for applying a carbazole-degrader, Pseudomonas sp. strain CA10, to dioxin-contaminated soil remediation. Appl. Microbiol. Biotechnol. 56:788-795.

Hassanov, T., Karunker, I., Steinberg, N., Erez, A., and Kolodkin-Gal, I. 2018. Novel antibiofilm chemotherapies target nitrogen from glutamate and glutamine. Sci. Rep. 8:7097.

Huang, X. F., Zhou, D., Guo, J., Manter, D. K., Reardon, K. F., and Vivanco, J. M. 2015. Bacillus spp. from rainforest soil promote plant growth under limited nitrogen conditions. J. Appl. Microbiol. 118:672-684.

Iven, T., König, S., Singh, S., Braus-Stromeyer, S. A., Bischoff, M., Tietze, L. F., Braus, G. H., Lipka, V., Feussner, I., and Dröge-Laser, W. 2012. Transcriptional activation and production of tryptophan-derived secondary metabolites in arabidopsis roots contributes to the defense against the fungal vascular pathogen Verticillium longisporum. Mol. Plant 5:1389-1402.

Kearns, D. B., Chu, F., Branda, S. S., Kolter, R., and Losick, R. 2005. A master regulator for biofilm formation by Bacillus subtilis. Mol. Microbiol. 55:739-749.

Keren-Paz, A., Brumfeld, V., Oppenheimer-Shaanan, Y., and Kolodkin-Gal, I. 2018. Micro-CT X-ray imaging exposes structured diffusion barriers within biofilms. NPJ Biofilms Microbiomes 4:8.

Kobayashi, K. 2008. S1rR/SlrA controls the initiation of biofilm formation in Bacillus subtilis. Mol. Microbiol. 69:1399-1410.

Kolodkin-Gal, I., Elsholz, A. K., Muth, C., Girguis, P. R., Kolter, R., and Losick, R. 2013. Respiration control of multicellularity in Bacillus subtilis by a complex of the cytochrome chain with a membraneembedded histidine kinase. Genes Dev. 27:887-899.

Kolter, R., and Greenberg, E. P. 2006. The superficial life of microbes. Nature 441:300-302.

Lee, J., Jayaraman, A., and Wood, T. K. 2007. Indole is an inter-species biofilm signal mediated by SdiA. BMC Microbiol. 7:42.

Lee, J. H., Kim, Y. G., Baek, K. H., Cho, M. H., and Lee, J. 2015. The multifaceted roles of the interspecies signalling molecule indole in Agrobacterium tumefaciens. Environ. Microbiol. 17:1234-1244.

Leo, A., Hansch, C., and Elkins, D. 1971. Partition coefficients and their uses. Chem. Rev. 71:525-616.

Li, X., Chopp, D. L., Russin, W. A., Brannon, P. T., Parsek, M. R., and Packman, A. I. 2015. Spatial patterns of carbonate biomineralization in biofilms. Appl. Environ. Microbiol. 81:7403-7410.
Li, X., Chopp, D. L., Russin, W. A., Brannon, P. T., Parsek, M. R., and Packman, A. I. 2016. In situ biomineralization and particle deposition distinctively mediate biofilm susceptibility to chlorine. Appl. Environ. Microbiol. 82:2886-2892.

Liu, J., Prindle, A., Humphries, J., Gabalda-Sagarra, M., Asally, M., Lee, D. Y., Ly, S., Garcia-Ojalvo, J., and Süel, G. M. 2015. Metabolic codependence gives rise to collective oscillations within biofilms. Nature 523:550-554.

Lombardía, E., Rovetto, A. J., Arabolaza, A. L., and Grau, R. R. 2006. A LuxS-dependent cell-to-cell language regulates social behavior and development in Bacillus subtilis. J. Bacteriol. 188:4442-4452.

López, D., Vlamakis, H., Losick, R., and Kolter, R. 2009. Paracrine signaling in a bacterium. Genes Dev. 23:1631-1638.

Marlow, V. L., Porter, M., Hobley, L., Kiley, T. B., Swedlow, J. R., Davidson, F. A., and Stanley-Wall, N. R. 2014. Phosphorylated DegU manipulates cell fate differentiation in the Bacillus subtilis biofilm. J. Bacteriol. 196:16-27.

McLoon, A. L., Kolodkin-Gal, I., Rubinstein, S. M., Kolter, R., and Losick, R. 2011. Spatial regulation of histidine kinases governing biofilm formation in Bacillus subtilis. J. Bacteriol. 193:679-685.

Mhatre, E., Sundaram, A., Hölscher, T., Mühlstädt, M., Bossert, J., and Kovács, A. T. 2017. Presence of calcium lowers the expansion of Bacillus subtilis colony biofilms. Microorganisms 5:5.

Mielich-Süss, B., and Lopez, D. 2015. Molecular mechanisms involved in Bacillus subtilis biofilm formation. Environ. Microbiol. 17: 555-565.

Mijakovic, I., Poncet, S., Boël, G., Mazé, A., Gillet, S., Jamet, E., Decottignies, P., Grangeasse, C., Doublet, P., Le Maréchal, P., and Deutscher, J. 2003. Transmembrane modulator-dependent bacterial tyrosine kinase activates UDP-glucose dehydrogenases. EMBO J. 22:4709-4718.

Mintz-Oron, S., Mandel, T., Rogachev, I., Feldberg, L., Lotan, O., Yativ, M., Wang, Z., Jetter, R., Venger, I., Adato, A., and Aharoni, A. 2008. Gene expression and metabolism in tomato fruit surface tissues. Plant Physiol. 147:823-851

Molina-Santiago, C., Daddaoua, A., Fillet, S., Duque, E., and Ramos, J. L. 2014. Interspecies signalling: Pseudomonas putida efflux pump TtgGHI is activated by indole to increase antibiotic resistance. Environ Microbiol. 16:1267-1281.

Ongena, M., and Jacques, P. 2008. Bacillus lipopeptides: Versatile weapons for plant disease biocontrol. Trends Microbiol. 16:115-125.

Oppenheimer-Shaanan, Y., Sibony-Nevo, O., Bloom-Ackermann, Z., Suissa, R., Steinberg, N., Kartvelishvily, E., Brumfeld, V., and Kolodkin-Gal, I 2016. Spatio-temporal assembly of functional mineral scaffolds within microbial biofilms. NPJ Biofilms Microbiomes 2:15031.

Pedras, M. S., Nycholat, C. M., Montaut, S., Xu, Y., and Khan, A. Q. 2002. Chemical defenses of crucifers: Elicitation and metabolism of phytoalexins and indole-3-acetonitrile in brown mustard and turnip. Phytochemistry 59:611-625.

Raaijmakers, J. M., De Bruijn, I., Nybroe, O., and Ongena, M. 2010. Natural functions of lipopeptides from Bacillus and Pseudomonas: More than surfactants and antibiotics. FEMS Microbiol. Rev. 34: 1037-1062.

Ren, D., Bedzyk, L. A., Ye, R. W., Thomas, S. M., and Wood, T. K. 2004. Stationary-phase quorum-sensing signals affect autoinducer-2 and gene expression in Escherichia coli. Appl. Environ. Microbiol. 70:2038-2043.

Ren, Y., Hansen, S. F., Ebert, B., Lau, J., and Scheller, H. V. 2014. Sitedirected mutagenesis of IRX9, IRX9L and IRX14 proteins involved in xylan biosynthesis: Glycosyltransferase activity is not required for IRX 9 function in Arabidopsis. PLoS One 9:e105014.

Romero, D., Aguilar, C., Losick, R., and Kolter, R. 2010. Amyloid fibers provide structural integrity to Bacillus subtilis biofilms. Proc. Natl. Acad. Sci. U.S.A. 107:2230-2234.

Rosenberg, G., Steinberg, N., Oppenheimer-Shaanan, Y., Olender, T., Doron, S., Ben-Ari, J., Sirota-Madi, A., Bloom-Ackermann, Z., and Kolodkin-Gal, I. 2016. Not so simple, not so subtle: The interspecies competition between Bacillus simplex and Bacillus subtilis and its impact on the evolution of biofilms. NPJ Biofilms Microbiomes 2: 15027.

Rubinstein, S. M., Kolodkin-Gal, I., McLoon, A., Chai, L., Kolter, R., Losick, R., and Weitz, D. A. 2012. Osmotic pressure can regulate matrix gene expression in Bacillus subtilis. Mol. Microbiol. 86:426436.

Rudrappa, T., Czymmek, K. J., Paré, P. W., and Bais, H. P. 2008. Rootsecreted malic acid recruits beneficial soil bacteria. Plant Physiol. 148 1547-1556

Sambrook, J., and Russell, D. W. 2001. Molecular Cloning: A Laboratory Manual. Cold Spring Harbor Laboratory Press, New York. 
Shemesh, M., and Chai, Y. 2013. A combination of glycerol and manganese promotes biofilm formation in Bacillus subtilis via histidine kinase KinD signaling. J. Bacteriol. 195:2747-2754.

Spaepen, S., Vanderleyden, J., and Remans, R. 2007. Indole-3-acetic acid in microbial and microorganism-plant signaling. FEMS Microbiol. Rev. $31: 425-448$.

Steinberg, N., and Kolodkin-Gal, I. 2015. The matrix reloaded: How sensing the extracellular matrix synchronizes bacterial communities. J. Bacteriol. 197:2092-2103.

Sugawara, S., Hishiyama, S., Jikumaru, Y., Hanada, A., Nishimura, T., Koshiba, T., Zhao, Y., Kamiya, Y., and Kasahara, H. 2009. Biochemical analyses of indole-3-acetaldoxime-dependent auxin biosynthesis in Arabidopsis. Proc. Natl. Acad. Sci. U.S.A. 106:5430-5435.

Tomberlin, J. K., Crippen, T. L., Wu, G., Griffin, A. S., Wood, T. K., and Kilner, R. M. 2017. Indole: An evolutionarily conserved influencer of behavior across kingdoms. BioEssays 39.

Townsley, L., Yannarell, S. M., Huynh, T. N., Woodward, J. J., and Shank, E. A. 2018. Cyclic di-AMP acts as an extracellular signal that impacts Bacillus subtilis biofilm formation and plant attachment. MBio 9:9.
Verhamme, D. T., Kiley, T. B., and Stanley-Wall, N. R. 2007. DegU coordinates multicellular behaviour exhibited by Bacillus subtilis. Mol. Microbiol. 65:554-568.

Verhamme, D. T., Murray, E. J., and Stanley-Wall, N. R. 2009. DegU and Spo0A jointly control transcription of two loci required for complex colony development by Bacillus subtilis. J. Bacteriol. 191: $100-108$.

Vlamakis, H., Chai, Y., Beauregard, P., Losick, R., and Kolter, R. 2013. Sticking together: Building a biofilm the Bacillus subtilis way. Nat. Rev. Microbiol. 11:157-168.

Wach, A. 1996. PCR-synthesis of marker cassettes with long flanking homology regions for gene disruptions in S. cerevisiae. Yeast 12:259-265.

Wilson, G. A., and Bott, K. F. 1968. Nutritional factors influencing the development of competence in the Bacillus subtilis transformation system. J. Bacteriol. 95:1439-1449.

Yona, A. H., Bloom-Ackermann, Z., Frumkin, I., Hanson-Smith, V., Charpak-Amikam, Y., Feng, Q., Boeke, J. D., Dahan, O., and Pilpel, Y 2013. tRNA genes rapidly change in evolution to meet novel translational demands. eLife 2:e01339. 